\title{
A New Version of the Exponentiated Exponential Distribution: Copula, Properties and Application to Relief and Survival Times
}

\author{
Hanaa Elgohari \\ Department of applied statistics, Faculty of commerce, Mansoura University, Egypt
}

\begin{abstract}
In this paper, we introduce a new generalization of the Exponentiated Exponential distribution. Various structural mathematical properties are derived. Numerical analysis for mean, variance, skewness and kurtosis and the dispersion index is performed. The new density can be right skewed and symmetric with "unimodal" and "bimodal" shapes. The new hazard function can be "constant", "decreasing", "increasing", "increasing-constant", "upsidedown-constant", "decreasingconstant". Many bivariate and multivariate type model have been also derived. We assess the performance of the maximum likelihood method graphically via the biases and mean squared errors. The usefulness and flexibility of the new distribution is illustrated by means of two real data sets.
\end{abstract}

Keywords Exponentiated Exponential; Morgenstern family; Clayton Copula; Real Data Modeling; Hazard Function.

AMS 2010 subject classifications 62N01; 62N02; 62E10

DOI: $10.19139 /$ soic-2310-5070-1093

\section{Introduction and motivation}

A random variable (RV) $W$ is said to have the Exponentiated Exponential (EE) distribution if its probability density function (PDF) is given by

$$
\left.\pi_{a, b}(\mathbf{w})\right|_{(\mathbf{w}>0, a>0 \text { and } b>0)}=a b \mathrm{e}^{-b \mathbf{w}}\left(1-\mathrm{e}^{-b \mathbf{w}}\right)^{a-1} .
$$

The corresponding cumulative distribution function (CDF) can be written as

$$
\left.\mathbb{W}_{a, b}(\mathbf{w})\right|_{(\mathbf{w}>0, a>0 \text { and } b>0)}=\left(1-\mathrm{e}^{-b \mathbf{w}}\right)^{a} .
$$

Clearly, for $a=1$, the EE reduces to the standard exponential (E) model. If $1>a$, the function $\pi_{a, b}(\mathbf{w})$ monotonically decreases with $\mathbf{w}$. If $a>1$, the function $\pi_{a, b}(\mathbf{w})$ attains a mode at $\mathbf{w}=\frac{1}{b} \log (a)$. The statistical properties of the EE model have been studied by many authors. Many authors have derived and studied the EE model, see Zheng [66], Zheng and Park [67], Kundu and Pradhan [48], Aslam et al. [15], Aryal et al. [13], Khalil et al. [42], Abouelmagd et al. ([1],[2]), Ibrahim et al. [35] and Bhatti et al. [16] among others. Recently, Alizadeh et al. [6] defined a new family based on the exponential model called the generalized odd generalized exponential family of distributions. Analogously, Hamedani et al. ([33] and [34]) defined the the type I and type I general exponential class of distributions. Other works can be cited such as Korkmaz et al. [46] (exponential Lindley odd log-logistic family) and Yadav et al. [62] (Burr-Hatke exponential model). In the work, we introduce a new version of the EE model using the Odd-Burr generalized (OB-G) family called the OBEE (OBEE). On the other hand, some new bivariate type OBEE are derived. Due to Alizadeh et al. [6], the CDF of the the OB-G family is given by

\footnotetext{
*Correspondence to: Hanaa Elgohari (Email: hanaa_elgohary@mans:edu:eg). Department of applied statistics, Faculty of commerce, Mansoura University, Egypt
}

ISSN 2310-5070 (online) ISSN 2311-004X (print)

Copyright (C) 2021 International Academic Press 


$$
F_{\alpha, \beta, \underline{\Phi}}(\mathbf{w})=1-\frac{\overline{\mathbb{W}}_{\underline{\Phi}}(\mathbf{w})^{\alpha \beta}}{\left[\mathbb{W}_{\underline{\Phi}}(\mathbf{w})^{\alpha}+\overline{\mathbb{W}}_{\underline{\Phi}}(\mathbf{w})^{\alpha}\right]^{\beta}},
$$

where $\overline{\mathbb{W}}_{\underline{\Phi}}(\mathbf{w})=1-\mathbb{W}_{\underline{\Phi}}(\mathbf{w})$ and $\underline{\Phi}$ refers to the parameter vector of the base line model. The PDF corresponding to (3) is given by

$$
f_{\alpha, \beta, \underline{\Phi}}(\mathbf{w})=\frac{\alpha \beta \pi_{\underline{\Phi}}(\mathbf{w}) \mathbb{W}_{\underline{\Phi}}(\mathbf{w})^{\alpha-1} \overline{\mathbb{W}}_{\underline{\Phi}}(\mathbf{w})^{\alpha \beta-1}}{\left[\mathbb{W}_{\underline{\Phi}}(\mathbf{w})^{\alpha}+\overline{\mathbb{W}}_{\underline{\Phi}}(\mathbf{w})^{\alpha}\right]^{1+\beta}},
$$

where $\pi_{\underline{\Phi}}(\mathbf{w})=d \mathbb{W}_{\underline{\Phi}}(\mathbf{w}) / d x$. For $\beta=1$, we get the Odd G (O-G) family. For $\alpha=1$, we have the proportional reversed hazard rate family (PRHR). The OBEE CDF is given by

$$
F_{\underline{\Lambda}}(\mathbf{w})=1-\frac{\left\{1-\left[1-\mathrm{e}^{-b \mathbf{w}}\right]^{a}\right\}^{\alpha \beta}}{\left(\left[1-\mathrm{e}^{-b \mathbf{w}}\right]^{a \alpha}+\left\{1-\left[1-\mathrm{e}^{-b \mathbf{w}}\right]^{a}\right\}^{\alpha}\right)^{\beta}},
$$

where $\underline{\Lambda}$ refers to the parameter vector of the new OBEE model. For $\beta=1$, the OBEE reduces to the OEE. For $\alpha=1$, the OBEE reduces to the PRHREE. The PDF corresponding to (5) is given by

$$
f_{\underline{\Lambda}}(\mathbf{w})=\alpha \beta a b \mathrm{e}^{-b \mathbf{w}} \frac{\left[1-\mathrm{e}^{-b \mathbf{w}}\right]^{\dot{a}}\left\{1-\left[1-\mathrm{e}^{-b \mathbf{w}}\right]^{a}\right\}^{\alpha \beta-1}}{\left(\left[1-\mathrm{e}^{-b \mathbf{w}}\right]^{a \alpha}+\left\{1-\left[1-\mathrm{e}^{-b \mathbf{w}}\right]^{a}\right\}^{\alpha}\right)^{1+\beta}},
$$

where $\dot{a}=a \alpha-1$. The hazard function (HRF) can be derived from $f_{\underline{\Lambda}}(\mathbf{w}) / S_{\underline{\Lambda}}(\mathbf{w})$. For simulation of this new model, we obtain the quantile function (QF) of $\mathbf{w}$ (by inverting (5)), say $\mathbf{w}_{u}=\bar{F}^{-1}(u)$, as

$$
\mathbf{w}_{u}=-b^{-1} \ln \left(1-\left\{\frac{\left[1-(1-u)^{\beta^{-1}}\right]^{\alpha^{-1}}}{(1-u)^{\frac{1}{\alpha \beta}}+\left[1-(1-u)^{\beta^{-1}}\right]^{\alpha^{-1}}}\right\}^{a^{-1}}\right) .
$$

Equation (7) is used for simulating the OBEE model. Figure 1 gives some PDF plots for some selected parameters value. Figure 2 gives some HRF plots for some selected parameters value. Based on Figure 1 the OBEE density can be right skewed and symmetric with unimodal and bimodal PDFs. Based on Figure 2 the OBEE HRF can be"constant", "decreasing", "increasing", "increasing-constant", "upside-downconstant" and "decreasingconstant".

\section{Mathematical properties}

\subsection{Useful representations}

Due to Alizadeh et al. (2016), the PDF in (6) can be expressed as

$$
f(\mathbf{w})=\sum_{\kappa=0}^{\infty} \nabla_{\kappa} \pi_{a^{*}, b}(\mathbf{w})
$$

where $a^{*}=a(1+\kappa)$ and

$$
\begin{aligned}
& \nabla_{\kappa}=\frac{\alpha \beta}{1+\kappa} \sum_{i_{1}, i_{2}=0}^{\infty} \sum_{i_{3}=\kappa}^{\infty}(-1)^{i_{2}+k+\kappa}\left(\begin{array}{c}
-(1+\beta) \\
i_{1}
\end{array}\right) \\
& \times\left(\begin{array}{c}
-\left[\alpha\left(1+i_{1}\right)+1\right] \\
i_{2}
\end{array}\right)\left(\begin{array}{c}
\alpha\left(1+i_{1}\right)+i_{2}+1 \\
i_{3}
\end{array}\right)\left(\begin{array}{c}
i_{3} \\
\kappa
\end{array}\right)
\end{aligned}
$$




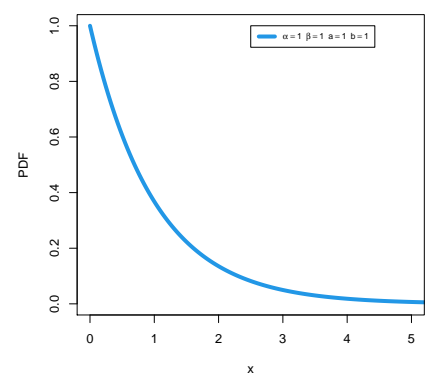

(a)

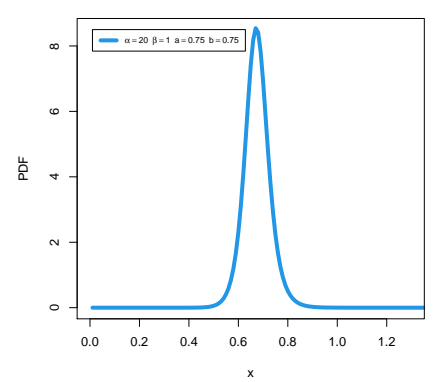

(d)

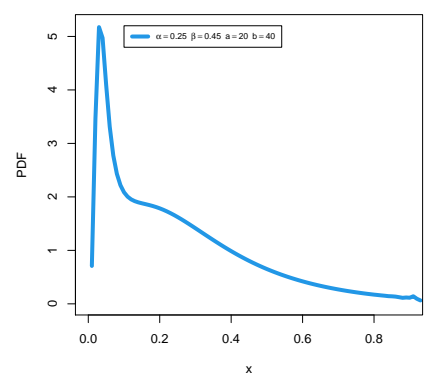

(b)

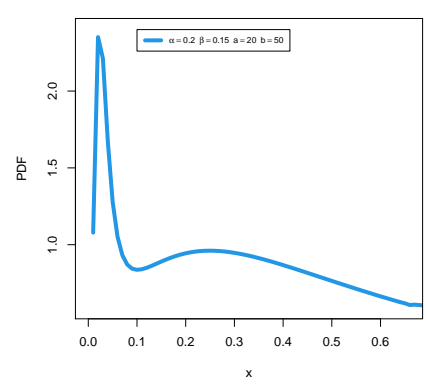

(e)

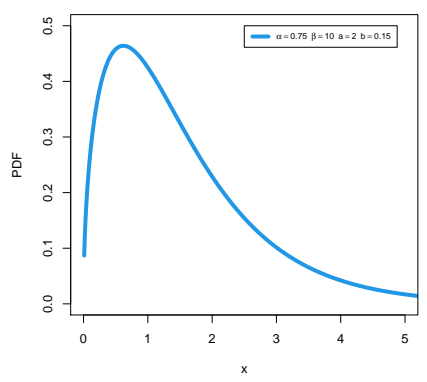

(c)

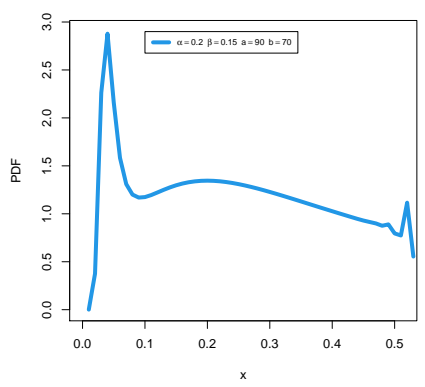

(f)

Figure 1. PDF plots for some selected parameters value.

and $\pi_{a^{*}, b}(\mathbf{w})$ refers to the density of the exponentiated exponential (EE) model with power parameter $a^{*}$. By integrating (8), the CDF of $W$ becomes

$$
F(\mathbf{w})=\sum_{\kappa=0}^{\infty} \nabla_{\kappa} \Pi_{a^{*}, b}(\mathbf{w}),
$$

where $\Pi_{a^{*}, b}(\mathbf{w})$ refers to the EE distribution with power parameter $a^{*}$.

\subsection{Asymptotics}

Let $c=\inf \left\{\left.F(\mathbf{w})\right|_{\mathbb{w}_{a, b}(\mathbf{w})>0}\right\}$, then

$$
\begin{aligned}
F_{\underline{\Lambda}}(\mathbf{w}) & \sim \beta\left(1-\mathrm{e}^{-b \mathbf{w}}\right)^{a \alpha} \text { as } \mathbf{w} \rightarrow 0, \\
f_{\underline{\Lambda}}(\mathbf{w}) & \sim \alpha \beta a b\left(1-\mathrm{e}^{-b \mathbf{w}}\right)^{\dot{a}} \mathrm{e}^{-b \mathbf{w}} \text { as } \mathbf{w} \rightarrow 0,
\end{aligned}
$$

and

$$
h_{\underline{\Lambda}}(\mathbf{w}) \sim \alpha \beta a b\left(1-\mathrm{e}^{-b \mathbf{w}}\right)^{\dot{a}} \mathrm{e}^{-b \mathbf{w}} \text { as } \mathbf{w} \rightarrow 0 .
$$

The asymptotics of CDF, PDF and HRF as $\mathbf{w} \rightarrow \infty$ are given by

$$
\begin{gathered}
1-F_{\underline{\Lambda}}(\mathbf{w}) \sim\left\{\alpha\left[1-\left(1-\mathrm{e}^{-b \mathbf{w}}\right)^{a}\right]\right\}^{\beta} \text { as } \mathbf{w} \rightarrow \infty, \\
f_{\underline{\Lambda}}(\mathbf{w}) \sim \frac{\alpha^{\beta} \beta a b\left(1-\mathrm{e}^{-b \mathbf{w}}\right)^{a-1} \mathrm{e}^{-b \mathbf{w}} \text { as } \mathbf{w} \rightarrow \infty,}{\left[1-\left(1-\mathrm{e}^{-b \mathbf{w}}\right)^{a}\right]^{1-\beta}},
\end{gathered}
$$




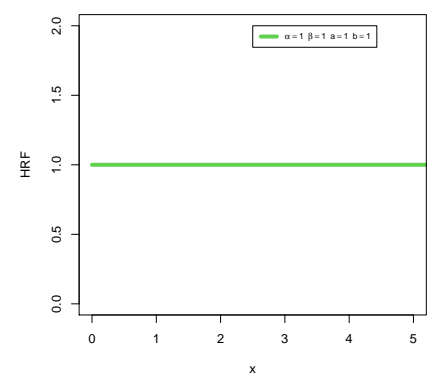

(a)

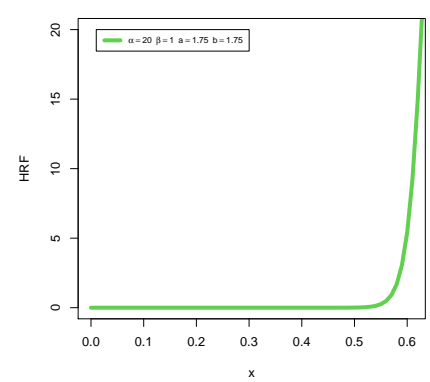

(d)

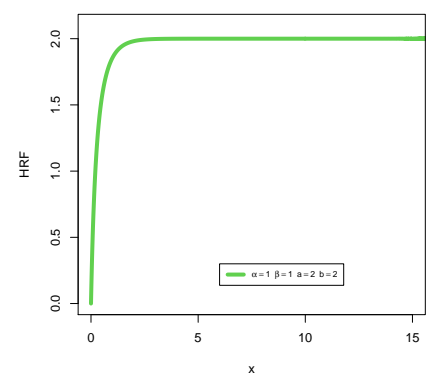

(g)

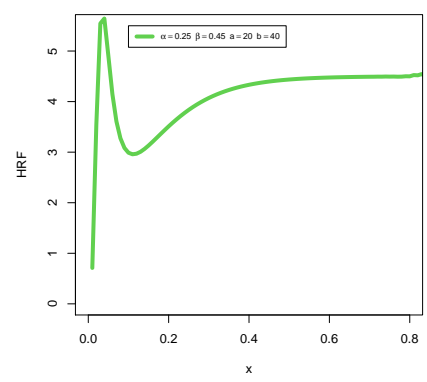

(b)

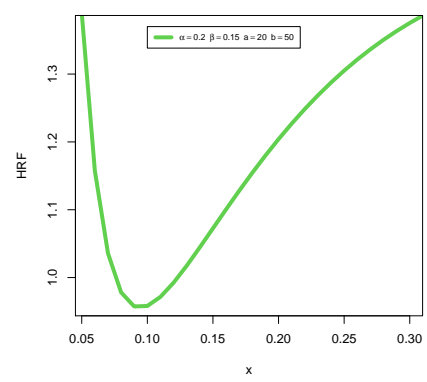

(e)

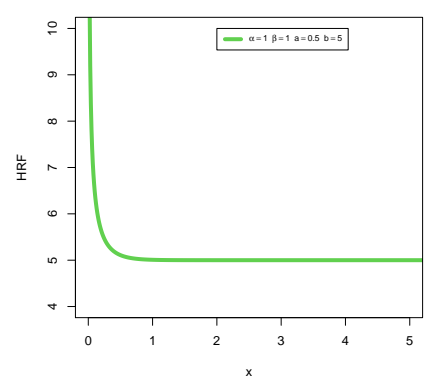

(h)

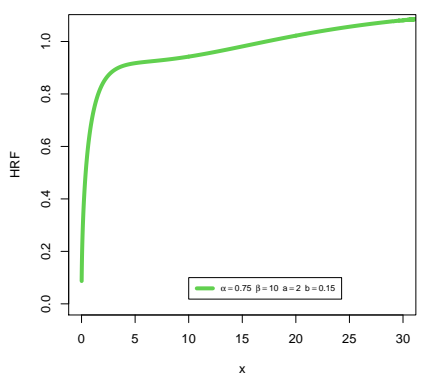

(c)

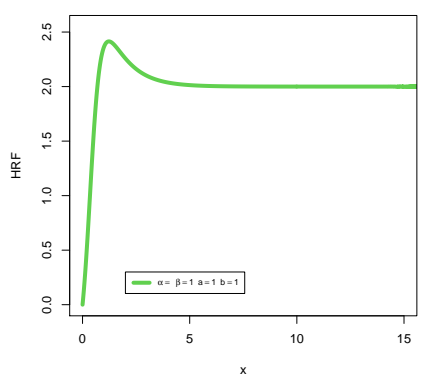

(f)

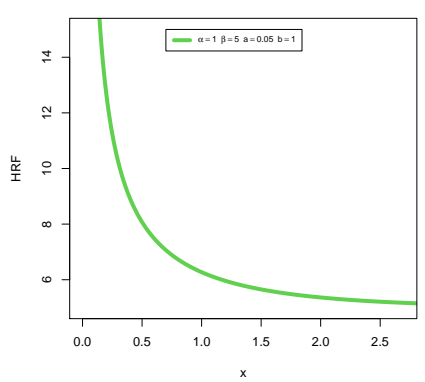

(i)

Figure 2. HDF plots for some selected parameters value.

and

$$
h_{\underline{\Lambda}}(\mathbf{w}) \sim \frac{\beta a b\left(1-\mathrm{e}^{-b \mathbf{w}}\right)^{a-1} \mathrm{e}^{-b \mathbf{w}}}{1-\left(1-\mathrm{e}^{-b \mathbf{w}}\right)^{a}} \text { as } \mathbf{w} \rightarrow \infty .
$$

\subsection{Moments and incomplete moments}

The $\varsigma^{\text {th }}$ ordinary moment of $W$ is given by

$$
\mu_{\varsigma}^{\prime}=\mathbf{E}\left(W^{\varsigma}\right)=\int_{-\infty}^{\infty} \mathbf{w}^{\varsigma} f(\mathbf{w}) d \mathbf{w}
$$


then we obtain

$$
\left.\mu_{\varsigma}^{\prime}\right|_{(\varsigma>-\mathbf{1})}=b^{-\varsigma} \Gamma(1+\varsigma) \sum_{\kappa, h=0}^{\infty} \nabla_{\kappa, h}^{\left(a^{*}, \varsigma\right)}
$$

where

$$
\nabla_{\kappa, h}^{\left(a^{*}, \varsigma\right)}=\nabla_{\kappa} \frac{a^{*}(-1)^{h}}{(h+1)^{\varsigma+1}}\left(\begin{array}{c}
a^{*}-1 \\
h
\end{array}\right)
$$

and

$$
\left.\Gamma(1+\zeta)\right|_{\left(\zeta \in \mathbf{R}^{+}\right)}=\zeta !=_{\zeta=0}^{\zeta-1}(\zeta-\varsigma) .
$$

where $\mathbf{E}(\mathbf{w})=\mu_{1}^{\prime}$ is the mean of $\mathbf{w}$. The $\varsigma^{t h}$ incomplete moment, say $\varphi_{\varsigma}(t)$, of $\mathbf{w}$ can be expressed, from (9), as

$$
\varphi_{\varsigma}(t)=\int_{-\infty}^{t} \mathbf{w}^{\varsigma} f(\mathbf{w}) d \mathbf{w}=\sum_{\kappa=0}^{\infty} \nabla_{\kappa} \int_{-\infty}^{t} \mathbf{w}^{\varsigma} \pi_{a^{*}, b}(\mathbf{w}) d \mathbf{w}
$$

then

$$
\left.\varphi_{\varsigma}(t)\right|_{(\varsigma>-\delta)}=b^{-\varsigma} \gamma(\varsigma+1, b t) \sum_{\kappa, h=0}^{\infty} \nabla_{\kappa, h}^{\left(a^{*}, \varsigma\right)},
$$

where $\gamma(\zeta, \vartheta)$ is the incomplete gamma function.

$$
\begin{aligned}
\left.\gamma(\zeta, \vartheta)\right|_{(\zeta \neq 0,-1,-2, \ldots)} & =\int_{0}^{\vartheta} \exp (-\mathbf{w}) d \mathbf{w} \\
& =\frac{1}{\zeta} \vartheta^{\zeta}\left\{{ }_{1} \mathbf{F}_{1}[\zeta ; \zeta+1 ;-\vartheta]\right\} \\
& =\sum_{\kappa=0}^{\infty} \frac{(-1)^{\kappa}}{\kappa !(\zeta+\kappa)} \vartheta^{\zeta+\kappa},
\end{aligned}
$$

and ${ }_{1} \mathbf{F}_{1}[\cdot, \cdot, \cdot]$ is a confluent hypergeometric function. The first incomplete moment given by (11) with $\varsigma=1$ as

$$
\varphi_{1}(t)=b \gamma\left(2, \frac{1}{t}\right) \sum_{\kappa, h=0}^{\infty} \nabla_{\kappa, h}^{\left(1, a^{*}\right)}
$$

\subsection{Moment generating function (MGF)}

The MGF $M_{W}(t)=\mathbf{E}(\exp (t W))$ of $W$ can be derived from equation (8) as

$$
M_{W}(t)=\sum_{\kappa=0}^{\infty} \nabla_{\kappa} M_{a^{*}, b}(T),
$$

where $M_{a^{*}, b}(T)$ is the MGF of the EW model with power parameter $a^{*}$.

$$
\left.M_{W}(t)\right|_{(\varsigma>-1)}=\sum_{\varsigma=0}^{\infty} \sum_{\kappa, h=0}^{\infty} \frac{t^{\varsigma}}{\varsigma !} b^{-\varsigma} \Gamma(1+\varsigma) \nabla_{\kappa, h}^{\left(a^{*}, \varsigma\right)} .
$$




\subsection{Residual life and reversed residual life functions}

The $\rho^{\text {th }}$ moment of the residual life

$$
a_{\rho}(t)=\mathbf{E}\left[\left.(Z-t)^{\rho}\right|_{\mathbf{w}>t, \rho=1,2, \ldots]} .\right.
$$

The $\rho^{\text {th }}$ moment of the residual life of $W$ is given by

$$
a_{\rho}(t)=\frac{1}{1-F_{\underline{\Lambda}}(t)} \int_{t}^{\infty}(\mathbf{w}-t)^{\rho} d F(\mathbf{w}) .
$$

Therefore,

$$
a_{\rho}(t)=\left.\frac{1}{1-F_{\underline{\Lambda}}(t)} \sum_{\kappa, h=0}^{\infty} \mathbf{c}_{\kappa, h}^{\left(a^{*}, \rho\right)} b^{\rho} \Gamma(\rho+1, b t)\right|_{(\rho>-1)},
$$

where

$$
\begin{gathered}
\mathbf{c}_{\kappa, h}^{\left(a^{*}, \rho\right)}=\nabla_{\kappa} \sum_{r=0}^{\rho}\left(\begin{array}{l}
\rho \\
r
\end{array}\right)(-t)^{\rho-\varsigma}, \\
\left.\Gamma(\zeta, \varsigma)\right|_{\varsigma>0}=\int_{\varsigma}^{\infty} \mathbf{w}^{\zeta-1} \exp (-\mathbf{w}) d \mathbf{w}
\end{gathered}
$$

and

$$
\Gamma(\zeta, \varsigma)=\Gamma(\zeta)-\gamma(\zeta, \varsigma)
$$

The $\rho^{\text {th }}$ moment of the reversed residual life, say

$$
A_{\rho}(t)=\mathbf{E}\left[\left.(t-Z)^{\rho}\right|_{\mathbf{w} \leq t, t>0 \text { and } \rho=1,2, \ldots}\right]
$$

uniquely determines $F_{\underline{\Lambda}}(\mathbf{w})$. We obtain

$$
A_{\rho}(t)=\frac{1}{F_{\underline{\Lambda}}(t)} \int_{0}^{t}(t-\mathbf{w})^{\rho} d F(\mathbf{w})
$$

Then, the $\rho^{t h}$ moment of the reversed residual life of $W$ becomes

$$
A_{\rho}(t)=\left.\frac{1}{F_{\underline{\boldsymbol{\Lambda}}}(t)} \sum_{\kappa, h=0}^{\infty} \mathbf{C}_{\kappa, h}^{\left(a^{*}, \rho\right)} b^{\rho} \gamma(\rho+1, b t)\right|_{(\varsigma>-\mathbf{1})},
$$

where

$$
\mathbf{C}_{\kappa, h}^{\left(a^{*}, \rho\right)}=\nabla_{\kappa} \sum_{r=0}^{\rho}(-1)^{\mathbf{r}}\left(\begin{array}{l}
\rho \\
r
\end{array}\right) t^{\rho-\mathbf{r}}
$$

\subsection{Numerical analysis}

Table 1 gives Numericals results for the variance $(\mathrm{V}(Z))$, mean $(\mathbf{E}(Z))$, kurtosis $(\mathrm{K}(Z))$, skewness $(\mathrm{S}(Z))$ and dispersion index $(\operatorname{DisIx}(Z))$. Based on Table 1, we note that: 1-The skewness of the OBEE distribution can range in the interval $(-2.7792,8.2978)$. 2-The spread for the OBEE kurtosis is much larger ranging from -46.275 to 35.526. 3-DisIx $(Z)$ can be "between 0 and 1" or "equal 1" or more than 1. 
Table 1: Mean, variance, skewness, kurtosis and dispersion index.

\begin{tabular}{|c|c|c|c|c|c|c|c|c|}
\hline$\overline{\alpha \alpha}$ & $\overline{\beta \beta}$ & $\bar{a}$ & 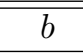 & $\overline{\overline{\mathbf{E}(Z)}}$ & 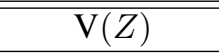 & $\overline{\overline{\mathrm{S}(Z)}}$ & 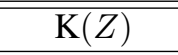 & $\overline{\text { DisIx }(Z)}$ \\
\hline 0.5 & 2 & 1.5 & 1.5 & 0.502266 & 0.4852427 & 2.478655 & 11.13074 & 0.9661068 \\
\hline 1 & & & & 0.4849408 & 0.1495599 & 1.598563 & 6.885345 & 0.3084085 \\
\hline 5 & & & & 0.5970219 & 0.0104331 & -0.0226027 & 3.506458 & 0.0174753 \\
\hline 20 & & & & 0.6444730 & 0.0007732 & -0.4386726 & 4.015127 & 0.0011998 \\
\hline 50 & & & & 0.6552973 & 0.0001281 & -0.5222116 & 4.205749 & 0.0001955 \\
\hline 100 & & & & 0.6590055 & $3.2397 \times 10^{-5}$ & -0.5496712 & 4.250314 & $4.9161 \times 10^{-5}$ \\
\hline 200 & & & & 0.6608784 & $8.1462 \times 10^{-6}$ & -0.5636244 & 4.324122 & $1.2326 \times 10^{-5}$ \\
\hline \multirow[t]{6}{*}{5} & 0.5 & 0.25 & 0.25 & 0.586532 & 0.40893890 & 4.073737 & 32.78264 & 0.6972151 \\
\hline & 1 & & & 0.3147107 & 0.05833588 & 2.792337 & 20.03788 & 0.1853635 \\
\hline & 10 & & & 0.08191879 & 0.00203066 & 0.665321 & 3.385543 & 0.0247887 \\
\hline & 50 & & & 0.03443559 & 0.00039684 & 0.678116 & 3.479092 & 0.0115240 \\
\hline & 100 & & & 0.02328808 & 0.00019240 & 0.515537 & 3.49165 & 0.0082615 \\
\hline & 200 & & & 0.01554562 & $9.0857 \times 10^{-5}$ & 1.290022 & 3.473409 & 0.0058446 \\
\hline \multirow[t]{9}{*}{2} & 5 & 0.5 & 5 & 0.019935 & 0.000259295 & 1.4310290 & 6.028403 & 0.01300695 \\
\hline & & 1 & & 0.068523 & 0.001137761 & 0.6051527 & 3.431216 & 0.01660408 \\
\hline & & 20 & & 0.553863 & 0.004671701 & -0.1685215 & 3.095139 & 0.00843472 \\
\hline & & 50 & & 0.733010 & 0.004878618 & -0.1930617 & 3.120336 & 0.00665559 \\
\hline & & 100 & & 0.870260 & 0.004949493 & -0.2013219 & 3.130186 & 0.00568737 \\
\hline & & 200 & & 1.008199 & 0.004985267 & -0.2054172 & 3.134844 & 0.00494473 \\
\hline & & 500 & & 1.191042 & 0.005006845 & -0.2078738 & 3.13767 & 0.00420375 \\
\hline & & 1000 & & 1.329533 & 0.005013977 & -0.2083907 & 3.132891 & 0.00377123 \\
\hline & & 2000 & & 1.468093 & 0.005017667 & -0.2091019 & 3.139091 & 0.00341781 \\
\hline \multirow[t]{7}{*}{1.5} & 1.5 & 1.5 & 0.5 & 1.7901830 & 1.055172 & 1.228803 & 5.744852 & 0.5894217 \\
\hline & & & 1 & 0.8950913 & 0.263793 & 1.228803 & 5.744886 & 0.2947109 \\
\hline & & & 5 & 0.1790183 & 0.010552 & 1.228803 & 5.744886 & 0.0589422 \\
\hline & & & 10 & 0.0895091 & 0.002638 & 1.228803 & 5.744886 & 0.0294711 \\
\hline & & & 50 & 0.0179018 & 0.0001055 & 1.113265 & 6.978685 & 0.0058942 \\
\hline & & & 100 & 0.0089509 & $2.638 \times 10^{-5}$ & 2.447838 & -2.87494 & 0.0029472 \\
\hline & & & 150 & 0.0059673 & $7.114 \times 10^{-6}$ & 9.999243 & -7.45278 & 0.0011922 \\
\hline 1 & 1 & 1 & 1 & 1 & 1 & 2 & 9 & 1 \\
\hline 2 & 2 & 2 & 100 & 0.0100978 & $1.3090 \times 10^{-5}$ & 22.320 & -168.8085 & 0.00129637 \\
\hline 2 & 2 & 2 & 500 & 0.0020196 & $1.0182 \times 10^{-5}$ & -1.2126 & 1.136435 & 0.00504159 \\
\hline 1 & 1 & 200 & 500 & 0.0117561 & $6.5598 \times 10^{-6}$ & 128.59 & -1726.776 & 0.00055799 \\
\hline
\end{tabular}

\section{Copula under the OBEE model}

In this section, we derive some new bivariate type OBEE (BvOBEE) model using FGM-copula, Clayton copula, modified FGM-copula and Renyi's entropy. The Multivariate OBEE (MvOBEE) type is also presented. Recently, many authors used and applied many different copulas in distribution theory such as Mansour et al. ([49], [50], [51], [52], [53],[54]), Elgohari and Yousof ([20], [21]), Salah et al. [?], Al-Babtain [3], Yousof et al. [64], Ibrahim et al. [39], Ali et al. ([4], [5]) and Yousof et al. [60]. 


\subsection{FGM copula}

First, we start with the joint CDF for Morgenstern family (Morgenstern (1956)) of two RVs $\left(W_{1}, W_{2}\right)$ which has the following form $C_{\lambda}(\varsigma, \omega)=(1+\lambda \bar{\varsigma} \omega) \varsigma \omega$ where $\lambda \in \mathbf{I}_{(-1,1)}$ and $\varsigma, \omega \in(0,1)$. Setting $\bar{\varsigma}=1-\varsigma$ and $\bar{\omega}=1-\omega$. Then,

$$
\left.\bar{\varsigma}\right|_{\left(\bar{\wp}_{\left(\mathbf{w}_{1}\right)}=1-\mathrm{e}^{\left.-b \mathbf{w}_{1}\right)}\right.}=\frac{\left\{1-\left[\bar{\wp}_{\left(\mathbf{w}_{1}\right)}\right]^{a}\right\}^{\alpha_{1} \beta_{1}}}{\left(\left[\bar{\wp}_{\left(\mathbf{w}_{1}\right)}\right]^{a \alpha_{1}}+\left\{1-\left[\bar{\wp}_{\left(\mathbf{w}_{1}\right)}\right]^{a}\right\}^{\alpha_{1}}\right)^{\beta_{1}}},
$$

where $a_{i}=\left.a\right|_{i=1,2}, b_{i}=\left.b\right|_{i=1,2}$. and

$$
\left.\bar{\omega}\right|_{\left(\bar{\wp}\left(\mathbf{w}_{2}\right)=1-\mathrm{e}^{\left.-b \mathbf{w}_{2}\right)}\right.}=\frac{\left\{1-\left[\bar{\wp}_{\left(\mathbf{w}_{2}\right)}\right]^{a}\right\}^{\alpha_{2} \beta_{2}}}{\left(\left[\bar{\wp}_{\left(\mathbf{w}_{2}\right)}\right]^{a \alpha_{2}}+\left\{1-\left[\bar{\wp}\left(\mathbf{w}_{2}\right)\right]^{a}\right\}^{\alpha_{2}}\right)^{\beta_{2}}},
$$

where $a_{i}=\left.a\right|_{i=1,2}, b_{i}=\left.b\right|_{i=1,2}$. Then, we have

$$
\begin{aligned}
& C_{\lambda}\left(\mathbf{w}_{1}, \mathbf{w}_{2}\right)=\left(1-\frac{\left\{1-\left[\bar{\wp}_{\left(\mathbf{w}_{1}\right)}\right]^{a}\right\}^{\alpha_{1} \beta_{1}}}{\left(\left[\bar{\wp}_{\left(\mathbf{w}_{1}\right)}\right]^{a \alpha_{1}}+\left\{1-\left[\bar{\wp}_{\left(\mathbf{w}_{1}\right)}\right]^{a}\right\}^{\alpha_{1}}\right)^{\beta_{1}}}\right) \\
& \times\left(1-\frac{\left\{1-\left[\bar{\wp}_{\left(\mathbf{w}_{2}\right)}\right]^{a}\right\}^{\alpha_{2} \beta_{2}}}{\left(\left[\bar{\wp}_{\left(\mathbf{w}_{2}\right)}\right]^{a \alpha_{2}}+\left\{1-\left[\bar{\wp}_{\left(\mathbf{w}_{2}\right)}\right]^{a}\right\}^{\alpha_{2}}\right)^{\beta_{2}}}\right)
\end{aligned}
$$

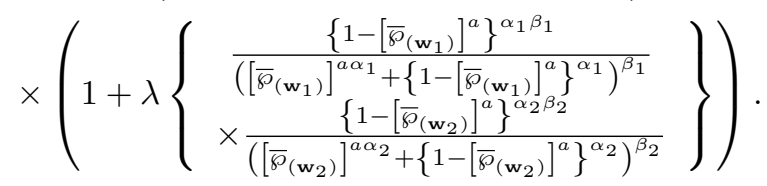

\subsection{Modified FGM copula}

Consider the following modified version of the bivariate FGM copula defined as (see Farlie [26], Gumbel [31], Gumbel [32] and Morgenstern [57])

$$
\left.C_{\boldsymbol{\Delta}}(\varsigma, \omega)\right|_{\boldsymbol{\Delta} \in(-1,1)}=\varsigma \omega[1+\boldsymbol{\Delta} \vartheta(\varsigma) \mho(\omega)]=\varsigma \omega+\Delta \dot{\vartheta}(\varsigma) \dot{\mho}(\omega)
$$

where $\dot{\vartheta}(\varsigma)=\varsigma \vartheta(\varsigma)$, and $\dot{\mho}(\omega)=\omega \mho(\omega)$. Where $\vartheta(\varsigma)$ and $\mho(\omega)$ are on $\mathbf{I}_{(0,1)}$ where $\vartheta(0)=\vartheta(1)=\mho(0)=$ $\mho(1)=0$. Let

$$
\begin{gathered}
\alpha=\inf \left\{\frac{\partial}{\partial \varsigma} \dot{\vartheta}(\varsigma): d_{1}(\varsigma)\right\}<0, \xi=\inf \left\{\frac{\partial}{\partial \omega} \dot{\mho}(\omega): d_{2}(\omega)\right\}>0, \\
\beta=\sup \left\{\frac{\partial}{\partial \varsigma} \dot{\vartheta}(\varsigma): d_{1}(\varsigma)\right\}<0, \eta=\sup \left\{\frac{\partial}{\partial \omega} \dot{\mho}(\omega): d_{2}(\omega)\right\}>0,
\end{gathered}
$$

and $\min (\alpha \beta, \xi \eta) \geq 1$ where

and

$$
\begin{gathered}
\frac{\partial}{\partial \varsigma} \dot{\vartheta}(\varsigma)=\vartheta(\varsigma)+\varsigma \frac{\partial}{\partial \varsigma} \vartheta(\varsigma), \\
d_{1}(\varsigma)=\left\{\varsigma: \varsigma \in \mathbf{I}_{(0,1)} \mid \frac{\partial}{\partial \varsigma} \dot{\vartheta}(\varsigma) \text { exists }\right\},
\end{gathered}
$$

$$
d_{2}(\omega)=\left\{\omega: \omega \in \mathbf{I}_{(0,1)} \mid \frac{\partial}{\partial \omega} \dot{\mho}(\omega) \text { exists }\right\} \text {. }
$$


3.2.1. Bivariate OBEE-FGM (Type-I) model The bivariate OBEE-FGM (Type-I) model

$$
\begin{aligned}
C_{\boldsymbol{\Delta}}(\varsigma, \omega)= & \boldsymbol{\Delta}[\dot{\vartheta}(\varsigma) \dot{\mho}(\omega)]+ \\
& \left\{\begin{array}{c}
{\left[1-\frac{\left\{1-\left[\bar{\gamma}_{(\varsigma)}\right]^{a}\right\}^{\alpha_{1} \beta_{1}}}{\left(\left[\bar{\gamma}_{(\varsigma)}\right]^{a \alpha_{1}}+\left\{1-\left[\bar{\gamma}_{(\varsigma)}\right]^{a}\right\}^{\alpha_{1}}\right)^{\beta_{1}}}\right]} \\
\times\left[1-\frac{\left\{1-\left[\bar{\gamma}_{(\omega)}\right]^{\alpha}\right\}^{\alpha_{1} \beta_{1}}}{\left(\left[\bar{\gamma}_{(\omega)}\right]^{a \alpha_{1}}+\left\{1-\left[\bar{\gamma}_{(\omega)}\right]^{a}\right\}^{\alpha_{1}}\right)^{\beta_{1}}}\right]
\end{array}\right\},
\end{aligned}
$$

where

$$
\dot{\vartheta}(\varsigma)=\varsigma \frac{\left\{1-\left[\bar{\wp}_{(\varsigma)}\right]^{a}\right\}^{\alpha_{1} \beta_{1}}}{\left(\left[\bar{\wp}_{(\varsigma)}\right]^{a \alpha_{1}}+\left\{1-\left[\bar{\wp}_{(\varsigma)}\right]^{a}\right\}^{\alpha_{1}}\right)^{\beta_{1}}},
$$

and

$$
\dot{\mho}(\omega)=\omega \frac{\left\{1-\left[\bar{\wp}_{(\omega)}\right]^{a}\right\}^{\alpha_{1} \beta_{1}}}{\left(\left[\bar{\wp}_{(\omega)}\right]^{a \alpha_{1}}+\left\{1-\left[\bar{\wp}_{(\omega)}\right]^{a}\right\}^{\alpha_{1}}\right)^{\beta_{1}}} .
$$

3.2.2. BivariateOBEE-FGM (Type II) model Consider $\vartheta(\varsigma)$ and $\mho(\omega)$ where

$$
\left.\vartheta^{*}(\varsigma)\right|_{\left(\boldsymbol{\Delta}_{1}>0\right)}=\varsigma^{\boldsymbol{\Delta}_{1}}(1-\varsigma)^{1-\boldsymbol{\Delta}_{1}} \text { and }\left.\mho^{*}(\omega)\right|_{\left(\boldsymbol{\Delta}_{2}>0\right)}=\omega^{\boldsymbol{\Delta}_{2}}(1-\omega)^{1-\boldsymbol{\Delta}_{2}} .
$$

The bivariate OBEE-FGM (Type-II) copula can be derived from

$$
C_{\boldsymbol{\Delta}, \boldsymbol{\Delta}_{1}, \boldsymbol{\Delta}_{2}}(\varsigma, \omega)=\varsigma \omega\left[1+\boldsymbol{\Delta} \vartheta^{*}(\varsigma) \mho^{*}(\omega)\right] .
$$

\subsection{The bivariate OBEE via Renyi's entropy}

Following Pougaza and Djafari [58], the joint CDF of the bivariate OBEE via Renyi's entropy can be written as

$$
C(\varsigma, \omega)=\mathbf{w}_{2} \varsigma+\mathbf{w}_{1} \omega-\mathbf{w}_{1} \mathbf{w}_{2},
$$

then, the associated bivariate OBEE will be

$$
\begin{aligned}
C\left(\mathbf{w}_{1}, \mathbf{w}_{2}\right)= & \mathbf{w}_{2}\left[1-\frac{\left\{1-\left[\bar{\wp}_{\left(\mathbf{w}_{1}\right)}\right]^{a}\right\}^{\alpha_{1} \beta_{1}}}{\left(\left[\bar{\wp}_{\left(\mathbf{w}_{1}\right)}\right]^{a \alpha_{1}}+\left\{1-\left[\bar{\wp}_{\left(\mathbf{w}_{1}\right)}\right]^{a}\right\}^{\alpha_{1}}\right)^{\beta_{1}}}\right] \\
+ & +\mathbf{w}_{1}\left[\frac{\left\{1-\left[\bar{\wp}_{\left(\mathbf{w}_{2}\right)}\right]^{a}\right\}^{\alpha_{2} \beta_{2}}}{\left(\left[\bar{\wp}_{\left(\mathbf{w}_{2}\right)}\right]^{a \alpha_{2}}+\left\{1-\left[\bar{\wp}_{\left(\mathbf{w}_{2}\right)}\right]^{a}\right\}^{\alpha_{2}}\right)^{\beta_{2}}}\right]-\mathbf{w}_{1} \mathbf{w}_{2},
\end{aligned}
$$

where $a_{1}=a_{2}=a, b_{1}=b_{2}=b$. Then, we get the BOBEE type distribution via Renyi's entropy.

3.3.1. The bivariate $\mathrm{OBEE}$ extension Via Clayton copula The bivariate extension via Clayton copula can be considered as a weighted version of the Clayton copula, which is of the form

$$
\left.C(\varsigma, \omega)\right|_{[\eta \geq 0]}=\left[\varsigma^{-\eta}+\omega^{-\eta}-1\right]^{-\frac{1}{\eta}} .
$$

Next, setting $\varsigma=1-\bar{\varsigma}=\varsigma(x) \in \mathbf{I}_{(0,1)}$ and $\omega=1-\bar{\omega}=\omega(y) \in \mathbf{I}_{(0,1)}$. Then, the associated CDF bivariate OBEE type distribution will be 


$$
C(\mathbf{w}, y)=\left\{\begin{array}{c}
{\left[1-\frac{\left\{1-\left[\bar{\gamma}_{(\mathbf{w})}\right]^{a}\right\}^{\alpha_{1} \beta_{1}}}{\left(\left[\bar{\gamma}_{(\mathbf{w})}\right]^{\alpha_{1}}+\left\{1-\left[\bar{\gamma}_{(\mathbf{w})}\right]^{a}\right\}^{\alpha_{1}}\right)^{\beta_{1}}}\right]^{-\eta}} \\
+\left[1-\frac{\left\{1-\left[\bar{\gamma}_{(\mathbf{y})}\right]^{a}\right\}^{\alpha_{2} \beta_{2}}}{\left(\left[\bar{\gamma}_{(\mathbf{y})}\right]^{\alpha \alpha_{2}}+\left\{1-\left[\bar{\gamma}_{(\mathbf{y})}\right]^{\alpha}\right\}^{\alpha_{2}}\right)^{\beta_{2}}}\right]^{-\eta}-1
\end{array}\right\}
$$

3.3.2. The Multivariate OBEE extension A straightforward $d$-dimensional extension from the above will be

$$
C\left(\mathbf{w}_{1}, \mathbf{w}_{2}, \cdots, \mathbf{w}_{d}\right)=\left\{\begin{array}{c}
\sum_{i=1}^{d}\left[1-\frac{\left\{1-\left[\bar{\gamma}\left(\mathbf{w}_{i}\right)\right]^{a}\right\}^{\alpha_{i} \beta_{i}}}{\left(\left[\bar{\S}\left(\mathbf{w}_{i}\right)\right]^{\alpha \alpha_{i}}+\left\{1-\left[\bar{\S}\left(\mathbf{w}_{i}\right)\right]^{a}\right\}^{\alpha_{i}}\right)^{\beta_{i}}}\right]^{-\eta} \\
+1-d
\end{array}\right\}^{-\frac{1}{\eta}} .
$$

\section{Maximum likelihood method}

For getting the maximum likelihood estimates (MLE) of the vector $\underline{\boldsymbol{\Lambda}}$, we have the log-likelihood $(\ell(\underline{\boldsymbol{\Lambda}}))$ function

$$
\begin{aligned}
\ell(\underline{\boldsymbol{\Lambda}}) \quad & =n \log (\alpha \beta a b)-b_{i=1}^{n} \mathbf{w}_{i}+(\dot{a})_{i=1}^{n} \log \left[\bar{\wp}_{\left(\mathbf{w}_{i}\right)}\right] \\
& +(\alpha \beta-1)_{i=1}^{n} \log \left\{1-\left[\bar{\wp}_{\left(\mathbf{w}_{i}\right)}\right]^{a}\right\} \\
& -(1+\beta)_{i=1}^{n} \log \left(\left[\bar{\wp}_{\left(\mathbf{w}_{i}\right)}\right]^{a \alpha}+\left\{1-\left[\bar{\wp}_{\left(\mathbf{w}_{i}\right)}\right]^{a}\right\}^{\alpha}\right)
\end{aligned}
$$

The components of the score vector

$$
\partial \ell / \partial \underline{\boldsymbol{\Lambda}}=\mathbf{U}(\underline{\boldsymbol{\Lambda}})=(\partial \ell / \partial \alpha, \partial \ell / \partial \beta, \partial \ell / \partial a, \partial \ell / \partial b)
$$

are available if needed. We can compute the maximum values of the unrestricted and restricted log-likelihoods to obtain likelihood ratio (LR) statistics for testing some sub-models of the OBEE distribution.

\section{Simulations}

In statistics, simulation is usually used for assessing the performance of a method, typically when there is a lack of theoretical background. In this section, we assess the performance of the maximum likelihood (ML) method. The assessment can be performed numerically or graphically. Graphically, we can perform the simulation experiments to assess of the finite sample behavior of the ML estimators (MLEs) via the biases and mean squared errors (MSEs). The following algorithm is considered for the assessment:

1. Using the inversion method, we we generate $\mathrm{N}=1000$ samples of size $n$ from the OBEE distribution using (7).

2. Compute the MLEs for the 1000 samples, say

$$
\left.\left(\widehat{\alpha_{\hbar}}, \widehat{\beta_{\hbar}}, \widehat{a_{\hbar}}, \widehat{b_{\hbar}}\right)\right|_{(\hbar=1,2, \ldots, 2000)},
$$

3. Compute the standard errors (StErs) of the MLEs for the 1000 samples, say

$$
\left.\left(S_{\widehat{\alpha_{\hbar}}}, S_{\widehat{\beta_{\hbar}}}, S_{\widehat{a_{\hbar}}}, S_{\widehat{b_{\hbar}}}\right)\right|_{(\hbar=1,2, \ldots, 2000)} .
$$

The StErs were computed by inverting the observed information matrix. 
4. Compute the biases and MSEs given for $\underline{\mathbf{V}}=(\alpha, \beta, a, b)$.

5. Repeated these steps for $n=50,100, \ldots, 300$ with $\alpha=1,2, . ., 100, \beta=1,2, . ., 100, a=1,2, . ., 100$ and $b=1,2, . ., 100$, so computing biases $\left(\operatorname{Bias}_{\mathbf{V}}(n)\right), M \operatorname{SEs}\left(\operatorname{MSE}_{\mathbf{V}}(n)\right)$ for $\alpha, \beta, a, b \forall n=50,100, \ldots, 300$ where

$$
\left.\operatorname{Bias}_{\underline{\Lambda}}(n)\right|_{\left(\underline{\boldsymbol{\Lambda}}=\nu, \theta, c_{2}, c_{1}\right)}=\frac{1}{2000} \sum_{\hbar=1}^{2000}\left(\underline{\hat{\boldsymbol{\Lambda}}}_{\hbar}-\underline{\mathbf{V}}\right),
$$

and

$$
\left.\operatorname{MSE}_{\underline{\Lambda}}(n)\right|_{\left(\boldsymbol{\Lambda}=\nu, \theta, c_{2}, c_{1}\right)}=\frac{1}{2000} \sum_{\hbar=1}^{2000}\left(\underline{\widehat{\Lambda}}_{\hbar}-\underline{\mathbf{V}}\right)^{2}
$$

Bias of $\alpha$

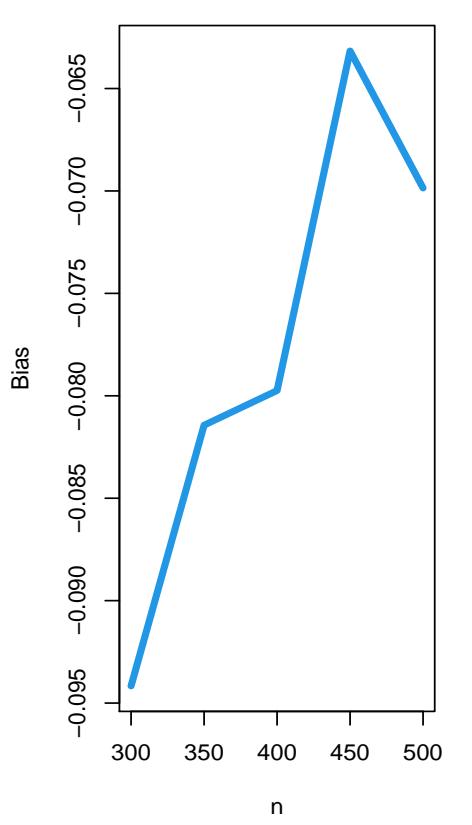

MSE of $\alpha$

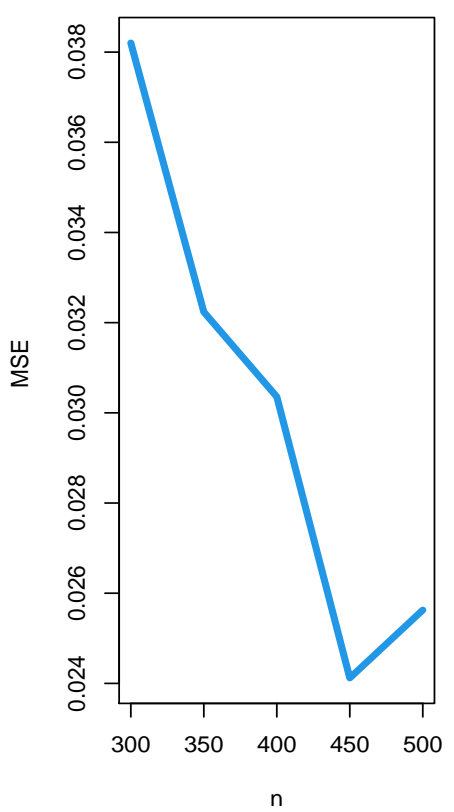

Figure 3. Biases (left) and MSEs (right) for the parameter .

Figures 3, 4, 5 and 6 gives the biases (left) and MSEs (right) for the parameters $\alpha, \beta, a$ and $b$ respectively. These figures (lefts) shows how the four biases vary with respect to $n$ and also shows how the four MSEs vary with respect to $n$. From Figures 3, 4, 5 and 6, the biases for each parameter are generally negative and getting close to zero as $n \rightarrow \infty$, the MSEs for each parameter decrease to zero as $n \rightarrow \infty$.

\section{Real data applications}

We shall compare thefits of the OBEE distribution with those of other competitive models, namely: Exponential (E), Odd Lindley Exponential (OLE), Marshall-Olkin Exponential (MOE), Moment Exponential (ME), The Logarithmic Burr-Hatke Exponential (LBHE), Generalized Marshall-Olkin Exponential (GMOE), Beta Exponential (BE), Marshall-Olkin Kumaraswamy Exponential (MOKwE), Kumaraswamy Exponential (KwE), the Burr X Exponential (BrXE) and Kumaraswamy Marshall-Olkin Exponential (KwMOE). Some other competitive model are can be derived based on Aryal, G. and Yousof 

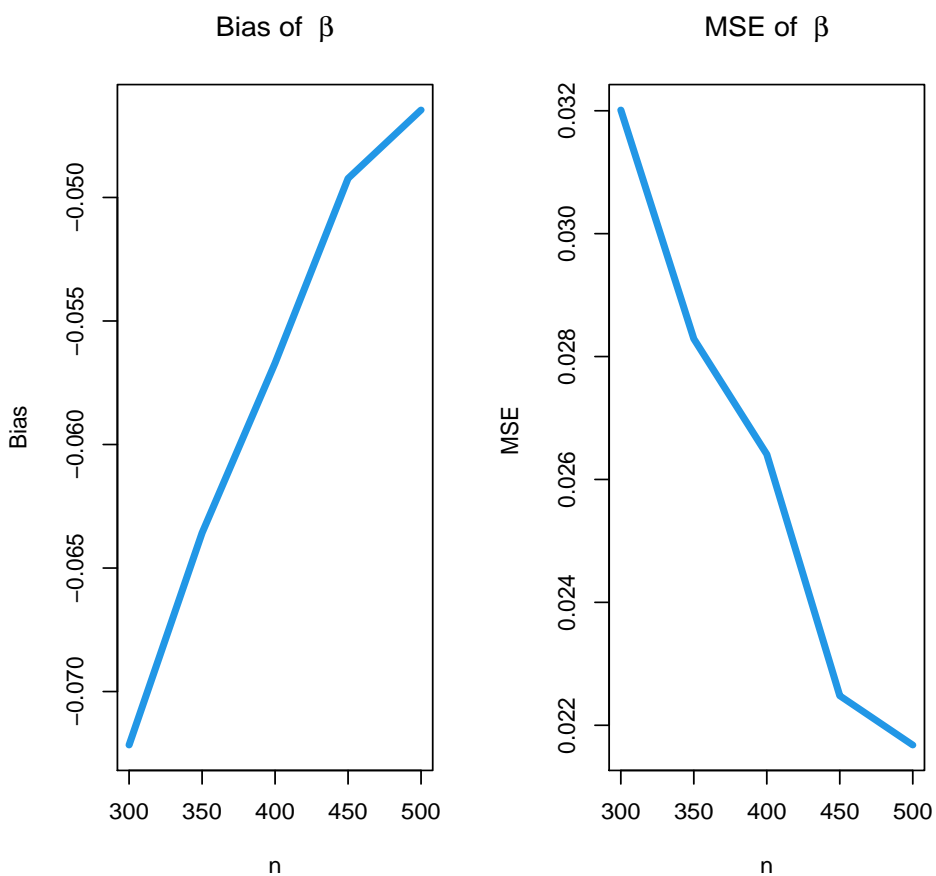

Figure 4. Biases (left) and MSEs (right) for the parameter .
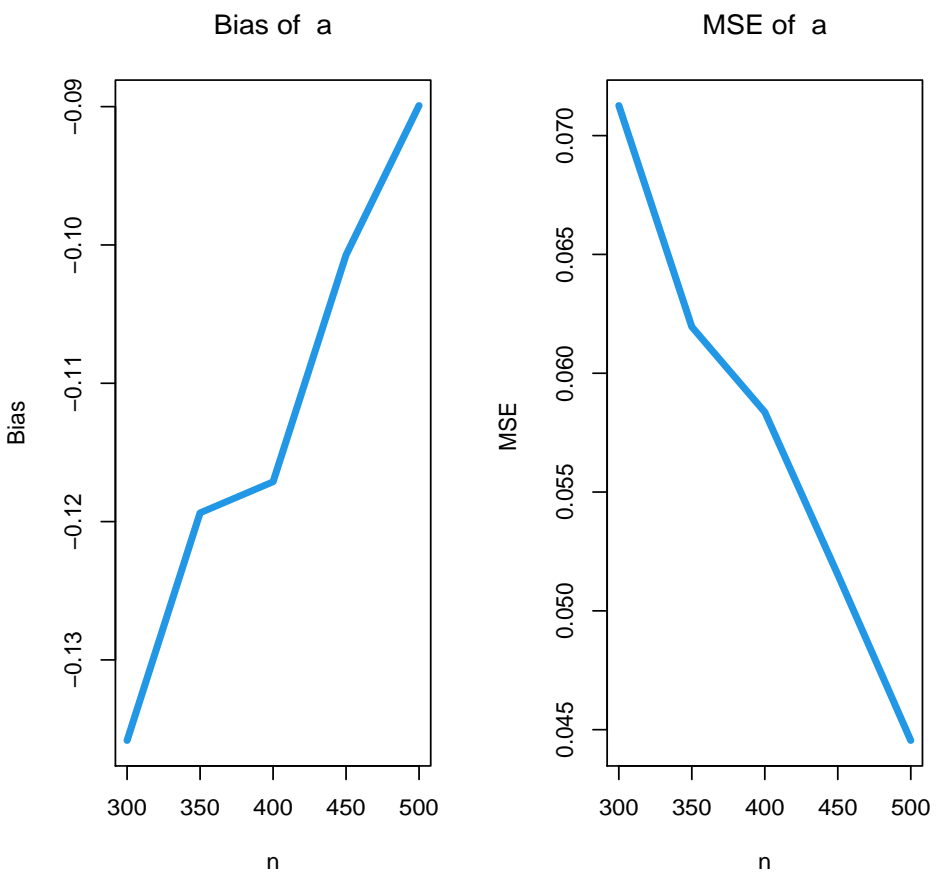

Figure 5. Biases (left) and MSEs (right) for the parameter a. 

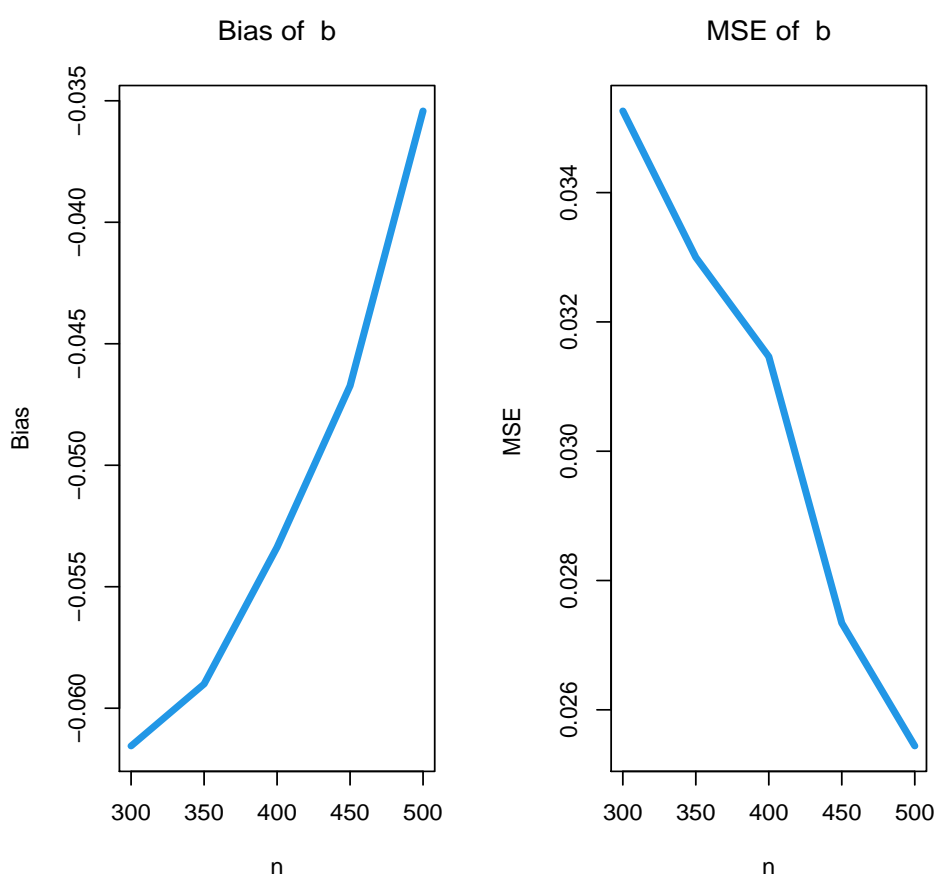

Figure 6. Biases (left) and MSEs (right) for the parameter b.

[14], Ibrahim et al. [36], Alizadeh et al. [8], Merovci et al. ([55], [59]), Korkmaz et al. [44], Karamikabir et al. [43] and Al-Babtain et al. [3]. For comparing models, we consider the Cramér-Von Mises (C) and the Anderson-Darling (A') and the Kolmogorov-Smirnov (KS) statistic. Moreover and for more accuracy, we consider another five goodness-of-fit measures: the Akaike Information Criterion (AIC) $\left(\mathrm{C}_{1}\right)$, Bayesian IC $\left(\mathrm{C}_{2}\right)$, Consistent AIC $\left(\mathrm{C}_{3}\right)$, Hannan-Quinn IC $\left(\mathrm{C}_{4}\right)$.

\subsection{Modeling lfaiure (relief) times}

The first data set $\{1.1,0.7,1.9,3.0,1.7,1.0,1.8,1.5,1.2,1.8,1.6,2.7,4.1,1.4,1.3,1.7,2.2,1.4,2.3,1.6$, 2 ) called the failure time data: The data represents the lifetime data relating to relief times (in minutes) of patients receiving an analgesic (see Gross and Clark [30]). This data was recently analyzed by Ibrahim et al. [40] and Al-Babtain et al. [3]. Table 2 lists the MLEs, StErs confidence intervals (CIs). Table 3 lists the $\mathrm{C}_{1}$, $\mathrm{C}_{2}, \mathrm{C}_{3}, \mathrm{C}_{4}, \mathrm{~A}, \mathrm{C}$, K.S. and p-value. Figure 7 gives the E-PDF, E-CDF, E-HRF and P-P plot for relief times data. Figure 8 below gives Kaplan-Meier survival plot for relief times data. 
Table 2: MLEs, StErs, CIs for the relief times data.

\begin{tabular}{|c|c|c|}
\hline Models & & MLEs, StErs and CIs \\
\hline \multirow{3}{*}{$\mathrm{E}_{(b)}$} & $\overline{\text { MLE }}$ & 0.526 \\
\hline & StEr & $(0.117)$ \\
\hline & $\mathrm{L}_{\mathrm{CI}}, \mathrm{U}_{\mathrm{CI}}$ & $(0.29,0.75)$ \\
\hline \multirow{3}{*}{$\mathrm{OLE}_{(b)}$} & MLE & 0.6044 \\
\hline & StEr & $(0.0535)$ \\
\hline & $\mathrm{L}_{\mathrm{CI}}, \mathrm{U}_{\mathrm{CI}}$ & $(0.5,0.7)$ \\
\hline \multirow{3}{*}{$\mathrm{ME}_{(b)}$} & MLE & 0.950 \\
\hline & StEr & $(0.150)$ \\
\hline & $\mathrm{L}_{\mathrm{CI}}, \mathrm{U}_{\mathrm{CI}}$ & $(0.66,1.24)$ \\
\hline \multirow{3}{*}{$\operatorname{LBHE}_{(b)}$} & MLE & 0.5263 \\
\hline & StEr & $(0.118)$ \\
\hline & $\mathrm{L}_{\mathrm{CI}}, \mathrm{U}_{\mathrm{CI}}$ & $(0.43,0.63)$ \\
\hline \multirow{3}{*}{$\operatorname{MOE}_{(\alpha, b)}$} & MLE & $54.474,2.316$ \\
\hline & StEr & $(35.582),(0.374)$ \\
\hline & $\mathrm{L}_{\mathrm{CI}}, \mathrm{U}_{\mathrm{CI}}$ & $(0,124.2),(1.58,3.05)$ \\
\hline \multirow{3}{*}{$\operatorname{GMOE}_{(\lambda, \alpha, b)}$} & MLE & $0.519,89.462,3.169$ \\
\hline & StEr & $(0.26),(66.28),(0.77)$ \\
\hline & $\mathrm{L}_{\mathrm{CI}}, \mathrm{U}_{\mathrm{CI}}$ & $(0.02,1),(0,219.4),(1.66,4.7)$ \\
\hline \multirow{3}{*}{$\mathrm{KwE}_{(\alpha, \beta, b)}$} & MLE & $83.756,0.568,3.330$ \\
\hline & StEr & $(42.361),(0.326),(1.188)$ \\
\hline & $\mathrm{L}_{\mathrm{CI}}, \mathrm{U}_{\mathrm{CI}}$ & $(0.73,166.78),(0,1.21),(1.00,5.66)$ \\
\hline \multirow{3}{*}{$\mathrm{BE}(\alpha, \beta, b)$} & MLE & $81.633,0.542,3.514$ \\
\hline & StEr & $(120.41),(0.33),(1.410)$ \\
\hline & $\mathrm{L}_{\mathrm{CI}}, \mathrm{U}_{\mathrm{CI}}$ & $(0,317.6),(0,1.2),(0.75,6.3)$ \\
\hline \multirow{3}{*}{$\operatorname{MOKwE}_{(\alpha, \beta, \lambda, b)}$} & MLE & $0.133,33.24,0.571,1.67$ \\
\hline & StEr & $(0.332),(57.85),(0.7),(1.8)$ \\
\hline & $\mathrm{L}_{\mathrm{CI}}, \mathrm{U}_{\mathrm{CI}}$ & $(0,0.8),(0,146.59),(0,1.98),(0,5.22)$ \\
\hline \multirow{3}{*}{$\operatorname{KwMOE}_{(\alpha, \beta, \lambda, b)}$} & MLE & $8.868,34.826,0.299,4.899$ \\
\hline & StEr & (9.146), (22.312), (0.239), (3.176) \\
\hline & $\mathrm{L}_{\mathrm{CI}}, \mathrm{U}_{\mathrm{CI}}$ & $(0,28.8),(0,78.6),(0,0.8),(0,11)$ \\
\hline \multirow{3}{*}{$\operatorname{BrXE}_{(a, b)}$} & MLE & $1.1635,0.3207$ \\
\hline & StEr & $(0.33),(0.03)$ \\
\hline & $\mathrm{L}_{\mathrm{CI}}, \mathrm{U}_{\mathrm{CI}}$ & $(0.5,1.8),(0.26,0.4)$ \\
\hline \multirow{3}{*}{$\mathbf{O B E E}_{(\alpha, \beta, a, b)}$} & MLE & $3.74,0.27,4.183,1.366$ \\
\hline & StEr & $(6.89),(0.19),(18.3),(2.94)$ \\
\hline & $\mathbf{L}_{\mathbf{C I}}, \mathbf{U}_{\mathbf{C I}}$ & $(0,16.9),(0,0.65),(0,40),(0,7.2)$ \\
\hline
\end{tabular}


Table 3: $\mathrm{C}_{1}, \mathrm{C}_{2}, \mathrm{C}_{3}, \mathrm{C}_{4}, \mathrm{~A}, \mathrm{C}$, K.S. and (p-value) for the relief times data.

\begin{tabular}{|c|c|c|c|c|}
\hline Models & $\mathrm{C}_{1}, \mathrm{C}_{2}, \mathrm{C}_{3}, \mathrm{C}_{4}$ & $\overline{\mathrm{A}}$ & $\mathrm{C}^{\cdot}$ & K.S. and (p-value) \\
\hline $\bar{E}$ & $67.70,68.70,67.89,68.90$ & 4.60 & 0.96 & $\begin{array}{c}0.44 \\
(<0.01)\end{array}$ \\
\hline OLE & $49.12,50.14,49.33,49.34$ & 1.3 & 0.22 & $\begin{array}{c}0.85 \\
(<0.001)\end{array}$ \\
\hline $\mathrm{ME}$ & $54.32,55.31,54.54,54.50$ & 2.76 & 0.53 & $\begin{array}{l}0.32 \\
(0.1)\end{array}$ \\
\hline LBHE & $67.70,68.70,67.89,67.90$ & 0.62 & 0.105 & $\begin{array}{c}0.44 \\
(<0.001)\end{array}$ \\
\hline MOE & $43.51,45.51,44.22,43.90$ & 0.8 & 0.14 & $\begin{array}{c}0.18 \\
(0.55)\end{array}$ \\
\hline GMOE & $42.75,45.74,44.25,43.34$ & 0.51 & 0.08 & $\begin{array}{c}0.15 \\
(0.78)\end{array}$ \\
\hline $\mathrm{KwE}$ & $41.78,44.75,43.28,42.32$ & 0.45 & 0.07 & $\begin{array}{c}0.14 \\
(0.86)\end{array}$ \\
\hline $\mathrm{BE}$ & $43.48,46.45,44.98,44.02$ & 0.70 & 0.12 & $\begin{array}{c}0.16 \\
(0.80)\end{array}$ \\
\hline MOKE & $41.58,45.54,44.25,42.30$ & 0.60 & 0.11 & $\begin{array}{c}0.14 \\
(0.87)\end{array}$ \\
\hline KMOE & $42.82,46.84,45.55,43.60$ & 1.08 & 0.19 & $\begin{array}{c}0.15 \\
(0.86)\end{array}$ \\
\hline $\mathrm{BrXE}$ & $48.13,50.15,48.83,48.52$ & 1.39 & 0.24 & $\begin{array}{c}0.248 \\
(0.171)\end{array}$ \\
\hline OBEE & $38.95,42.93,41.62,39.73$ & 0.155 & 0.0268 & $\begin{array}{c}0.0902 \\
(0.9969)\end{array}$ \\
\hline
\end{tabular}

Based on Table 3, we conclude that the proposed lifetime OBEE model is much better than all other mentioned models with $\mathrm{C}_{1}=38.95, \mathrm{C}_{2}=42.93, \mathrm{C}_{3}=41.62, \mathrm{C}_{4}=39.73, \mathrm{~A}=0.155, \mathrm{C}=0.0268$, K.S $=0.09016$ and $p$-value $=0.9969$ so the new lifetime model is a good alternative to these models in modeling relief times data set. According to Figures 8 and 9, the OBEE distribution provides adequate fits to the empirical functions.

\subsection{Modeling survival times}

The second data set $\{0.10,0.92,0.93,0.96,0.33,0.44,0.56,0.72,0.74,0.77,1.0,1.0,1.02,1.05,1.07$, $07,1.63,1.63,1.68,1.71,1.72,1.76,2.22,2.3,2.31,2.4,0.59,1.08,1.08,1.08,1.2,1.21,1.6,1.09,1.12$, $1.13,1.22,1.22,1.24,1.30,1.34,1.36,1.39,1.44,1.83,1.95,1.96,1.97,2.02,1.15,1.16,2.45,2.51,2.53$, $2.54,2.54,2.78,2.93,3.27,3.42,3.47,2.13,2.15,2.16,1.46,1.53,1.59,3.61,4.02,4.32,4.58,5.55\}$ called 
Box Plot

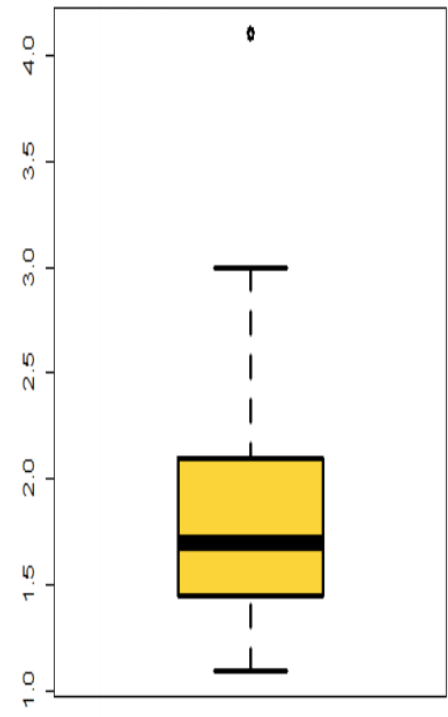

The Relief Times Data

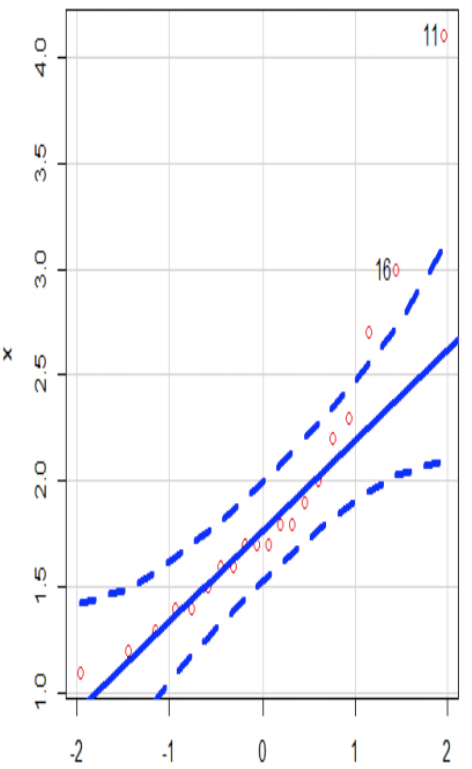

nom quantiles

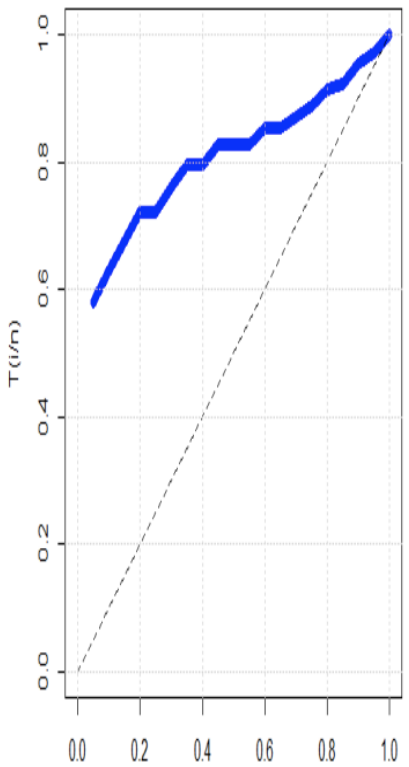

in

Figure 7. The box plot, Q-Q plot and TTT plot for the relief times data.

E.CDF for Relief Times Data
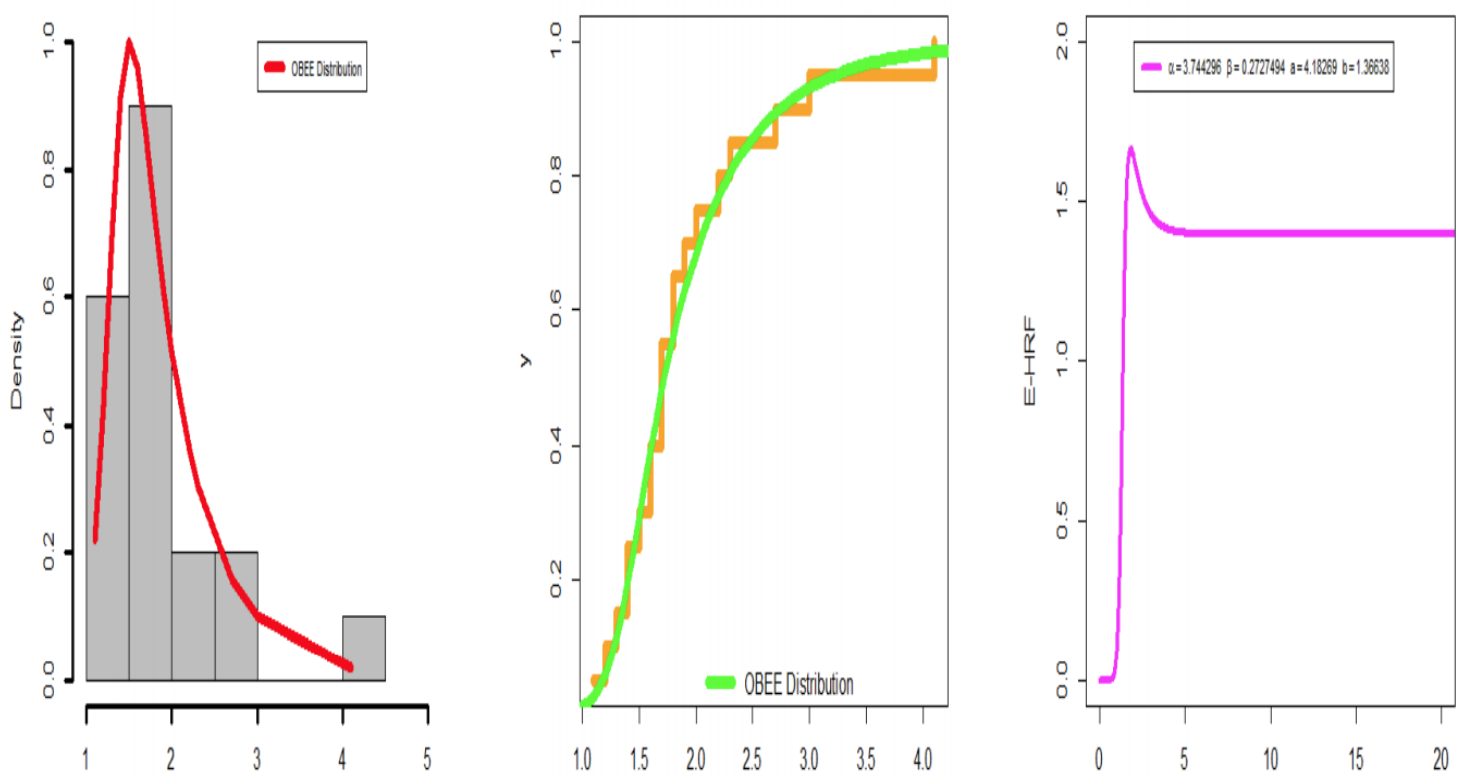

Relef Times Data

Figure 8. E-PDF, E-CDF and E-HRF for relief times data. 

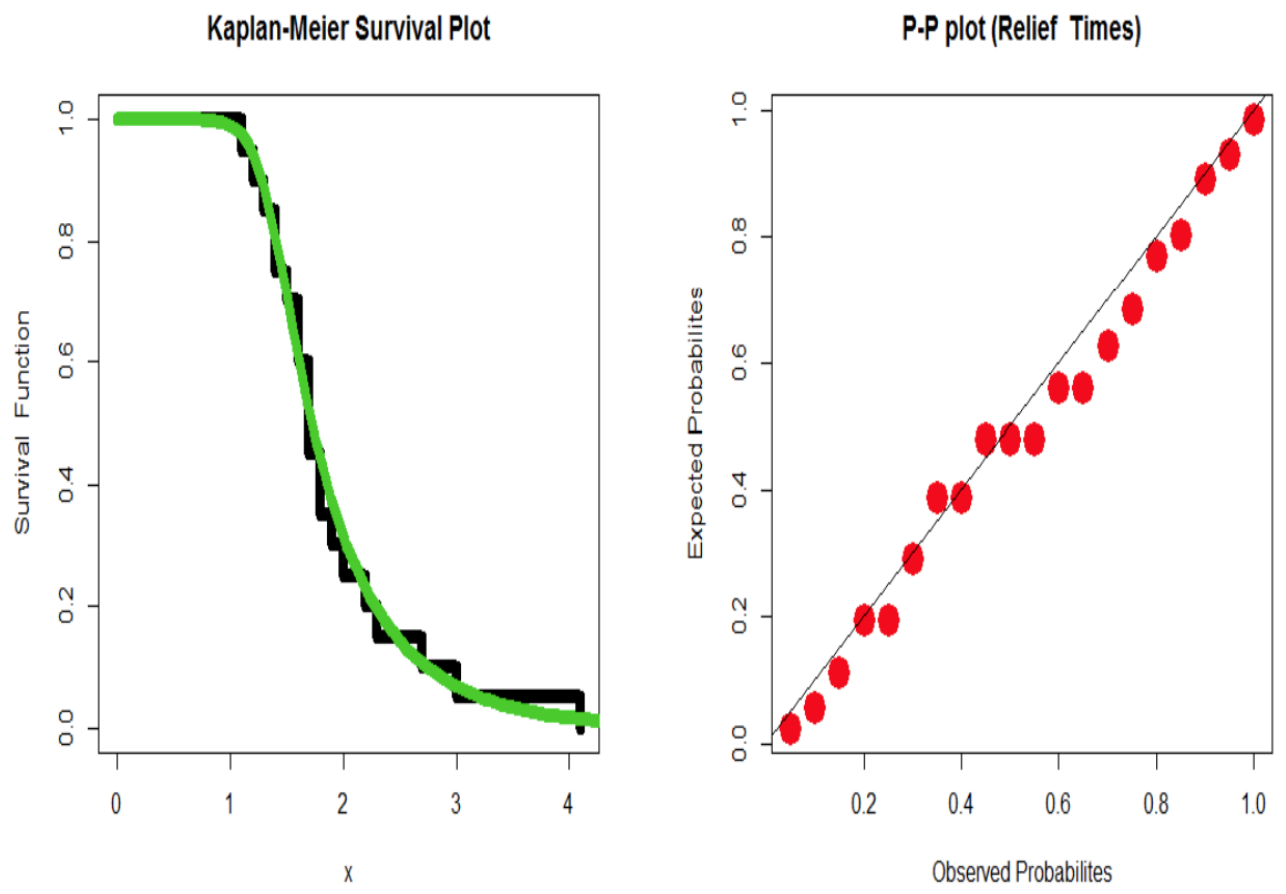

Figure 9. Kaplan-Meier survival plot and P-P plot for relief times data.

the survival times (in days) of 72 guinea pigs infected with virulent tubercle bacilli, observed and reported by Bjerkedal [17]. Table 4 lists the MLEs, StErs confidence intervals (CIs). Table 5 lists the $\mathrm{C}_{1}, \mathrm{C}_{2}, \mathrm{C}_{3}$, $\mathrm{C}_{4}, \mathrm{~A}, \mathrm{C}, \mathrm{K} . \mathrm{S}$. and p-value. For many other real-life data sets see [19], [22], [18], [47], [45], [63], [23], [24], [25], [65], [38], [61], [37], [7]. Figure 9 gives the estimated PDF (E-PDF), E-CDF, E-HRF and P-P plot survival times data. Figure 10 gives the Kaplan-Meier survival plot survival times data. Based on Table 5 , we conclude that the OBEE model is much better than all other competitive models with $\mathrm{C}_{1}=203.84$, $\mathrm{C}_{2}=212.94, \mathrm{C}_{3}=204.44, \mathrm{C}_{4}=207.46, \mathrm{~A}^{*}=0.297, \mathrm{C} \cdot=0.046, \mathrm{~K} . \mathrm{S}=0.0675$ and $\mathrm{p}$-value $=0.8987$ so the new lifetime model is a good alternative to these models in modeling relief times data set. According to Figures 11 and 12, the OBEE distribution provides adequate fits to the empirical functions. 
Table 4: MLEs, StErs, CIs for the survival times data.

\begin{tabular}{|c|c|c|}
\hline Models & & MLEs, StErs and CIs \\
\hline \multirow{3}{*}{$\mathrm{E}_{(b)}$} & MLE & 0.540 \\
\hline & $\mathrm{StEr}$ & $(0.063)$ \\
\hline & $\mathrm{L}_{\mathrm{CI}}, \mathrm{U}_{\mathrm{CI}}$ & $(0.4,0.7)$ \\
\hline \multirow{3}{*}{$\mathrm{OLE}_{(b)}$} & MLE & 0.3815 \\
\hline & StEr & $(0.021)$ \\
\hline & $\mathrm{L}_{\mathrm{CI}}, \mathrm{U}_{\mathrm{CI}}$ & $(0.3,0.4)$ \\
\hline \multirow{3}{*}{$\operatorname{ME}_{(b)}$} & MLE & 0.925 \\
\hline & $\mathrm{StEr}$ & $(0.077)$ \\
\hline & $\mathrm{L}_{\mathrm{CI}}, \mathrm{U}_{\mathrm{CI}}$ & $(0.6,1.1)$ \\
\hline \multirow{3}{*}{$\operatorname{LBHE}_{(b)}$} & MLE & 0.54 \\
\hline & $\mathrm{StEr}$ & $(0.064)$ \\
\hline & $\mathrm{L}_{\mathrm{CI}}, \mathrm{U}_{\mathrm{CI}}$ & $(0.4,0.67)$ \\
\hline \multirow{3}{*}{$\operatorname{MOE}_{(\alpha, b)}$} & MLE & $8.78,1.38$ \\
\hline & StEr & $(3.56),(0.19)$ \\
\hline & $\mathrm{L}_{\mathrm{CI}}, \mathrm{U}_{\mathrm{CI}}$ & $(1.8,15.7),(1.00,1.8)$ \\
\hline \multirow{3}{*}{$\operatorname{GMOE}_{(\lambda, \alpha, b)}$} & MLE & $0.18,47.64,4.47$ \\
\hline & StEr & $(0.07),(44.9),(1.33)$ \\
\hline & $\mathrm{L}_{\mathrm{CI}}, \mathrm{U}_{\mathrm{CI}}$ & $(0.04,0.3),(0,136),(1.8 .7)$ \\
\hline \multirow{3}{*}{$\operatorname{KwE}_{(a, \beta, b)}$} & MLE & $3.304,1.100,1.037$ \\
\hline & StEr & (1.106), (0.764), (0.614) \\
\hline & $\mathrm{L}_{\mathrm{CI}}, \mathrm{U}_{\mathrm{CI}}$ & $(1.13,5.47),(0,2.59),(0,2.24)$ \\
\hline \multirow{3}{*}{$\mathrm{BE}_{(a, \beta, b)}$} & MLE & $0.807,3.461,1.331$ \\
\hline & StEr & $(0.696),(1.003),(0.860)$ \\
\hline & $\mathrm{L}_{\mathrm{CI}}, \mathrm{U}_{\mathrm{CI}}$ & $(0,2.2),(1.49,5.42),(0,3)$ \\
\hline \multirow{3}{*}{$\operatorname{MOKwE}_{(\alpha, \beta, \lambda, b)}$} & MLE & $0.008,2.716,1.986,0.099$ \\
\hline & StEr & $(0.002),(1.316),(0.784),(0.05)$ \\
\hline & $\mathrm{L}_{\mathrm{CI}}, \mathrm{U}_{\mathrm{CI}}$ & $(0.004,0.01),(0.14,5),(0.5,4),(0,0.2)$ \\
\hline \multirow{3}{*}{$\operatorname{KwMOE}_{(\alpha, \beta, \lambda, b)}$} & MLE & $0.373,3.478,3.306,0.299$ \\
\hline & StEr & $(0.136),(0.861),(0.779),(1.112)$ \\
\hline & $\mathrm{L}_{\mathrm{CI}}, \mathrm{U}_{\mathrm{CI}}$ & $(0.11,0.6),(1.79,5),(1.78,5),(0,3)$ \\
\hline \multirow{3}{*}{$\operatorname{BrXE}_{(a, b)}$} & MLE & $0.48,0.21$ \\
\hline & StEr & $(0.060),(0.012)$ \\
\hline & $\mathrm{L}_{\mathrm{CI}}, \mathrm{U}_{\mathrm{CI}}$ & $(0.36,0.49),(0.18,0.23)$ \\
\hline \multirow{3}{*}{$\mathbf{O B E E}_{(\alpha, \beta, a, b)}$} & MLE & $3.5,0.59,0.56,0.28$ \\
\hline & StEr & $3.138,0.306,0.711,0.557$ \\
\hline & $\mathbf{L}_{\mathbf{C I}}, \mathbf{U}_{\mathbf{C I}}$ & $(0,7.9),(0,1.21),(0,1.96),(0,1.48)$ \\
\hline
\end{tabular}


Table 5: $\mathrm{C}_{1}, \mathrm{C}_{2}, \mathrm{C}_{3}, \mathrm{C}_{4}, \mathrm{~A}, \mathrm{C}, \mathrm{K} . \mathrm{S}$. and (p-value) for survival times data.

\begin{tabular}{|c|c|c|c|c|}
\hline Models & $\mathrm{C}_{1}, \mathrm{C}_{2}, \mathrm{C}_{3}, \mathrm{C}_{4}$ & $\overline{\mathrm{A}}$ & $\mathrm{C} \cdot$ & K.S. and (p-value) \\
\hline$\overline{\mathrm{E}}$ & $234.60,236.90,234.68,235.55$ & 6.53 & 1.25 & $\begin{array}{c}0.3 \\
(0.06)\end{array}$ \\
\hline OLE & $229.13,231.43,229.21,230.11$ & 1.94 & 0.33 & $\begin{array}{c}0.5 \\
(<0.001)\end{array}$ \\
\hline ME & $210.40,212.68,210.45,211.30$ & 1.52 & 0.25 & $\begin{array}{c}0.15 \\
(0.13)\end{array}$ \\
\hline LBHE & $234.63,236.92,234.71,235.51$ & 0.71 & 0.115 & $\begin{array}{c}0.28 \\
(<0.001)\end{array}$ \\
\hline MOE & $210.37,214.93,210.52,212.17$ & 1.18 & 0.17 & $\begin{array}{c}0.10 \\
(0.43)\end{array}$ \\
\hline GMOE & $210.54,217.38,210.89,213.24$ & 1.02 & 0.16 & $\begin{array}{l}0.09 \\
(0.5)\end{array}$ \\
\hline KwE & $209.42,216.24,209.77,212.12$ & 0.74 & 0.11 & $\begin{array}{l}0.09 \\
(0.5)\end{array}$ \\
\hline $\mathrm{BE}$ & 207.37, 214.21, 207.73, 210.09, & 0.98 & 0.15 & $\begin{array}{c}0.11 \\
(0.34)\end{array}$ \\
\hline MOKwE & $209.44,218.56,210.04,213.04$ & 0.79 & 0.12 & $\begin{array}{c}0.10 \\
(0.44)\end{array}$ \\
\hline KwMOE & $207.82,216.94,208.42,211.42$ & 0.61 & 0.11 & $\begin{array}{l}0.09 \\
(0.5)\end{array}$ \\
\hline $\mathrm{BrXE}$ & $235.31,239.92,235.53,237.14$ & 2.9 & 0.52 & $\begin{array}{c}0.22 \\
(0.002)\end{array}$ \\
\hline OBEE & $203.84,212.94,204.44,207.46$ & 0.297 & 0.0460 & $\begin{array}{c}0.0675 \\
(0.8987)\end{array}$ \\
\hline
\end{tabular}

\section{7. conclusions}

In this article, we introduced and studied a new flexible version of the exponentiated exponential model called the odd Burr exponentiated exponential (OBEE) model. The new density can be right skewed and symmetric with unimodal and bimodal shapes. The new HRF can be "constant", "decreasing", "increasing", "increasingconstant", "upside-down-constant", "decreasing-constant". Some of its mathematical properties including the ordinary moments, incomplete moment, moment generating function are derived. Numerical calculations for the expected value, skewness, variance, kurtosis and the index of dispersion is presented. The skewness of the OBEE distribution can range in the interval $(-2.779,8.2978)$. The spread for the OBEE kurtosis is much larger ranging from -46.275 to 35.526 . The index of dispersion can be "between 0 and 1" or "equal 


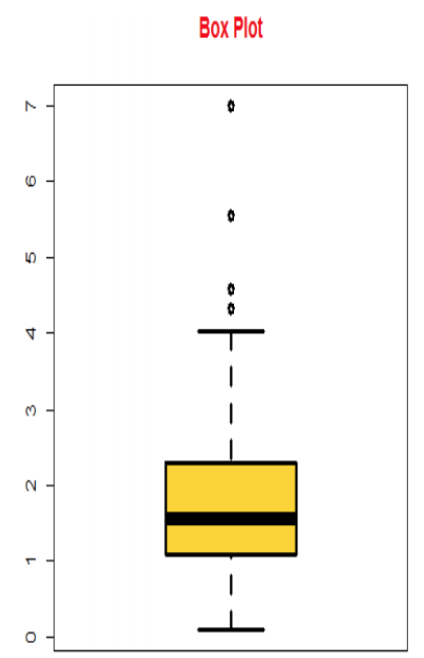

The Sunvival Times Data

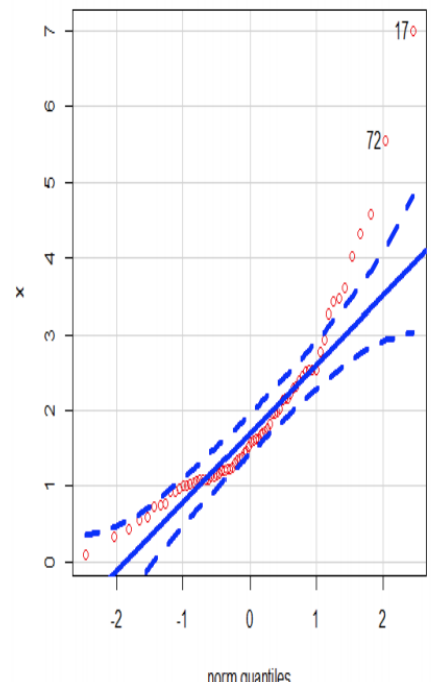

norm quartiles

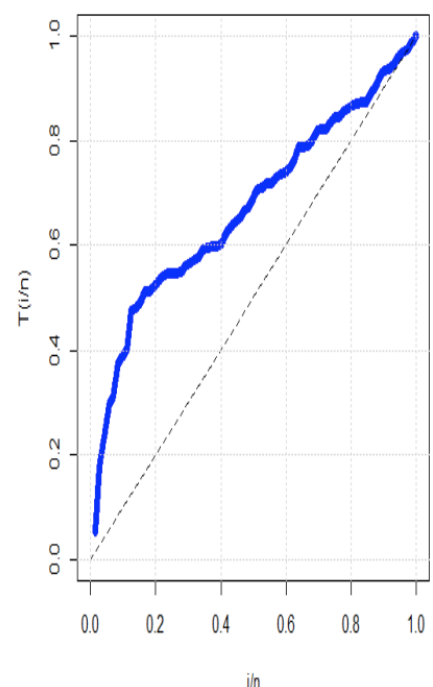

Figure 10. The box plot, Q-Q plot and TTT plot for the survival times data.
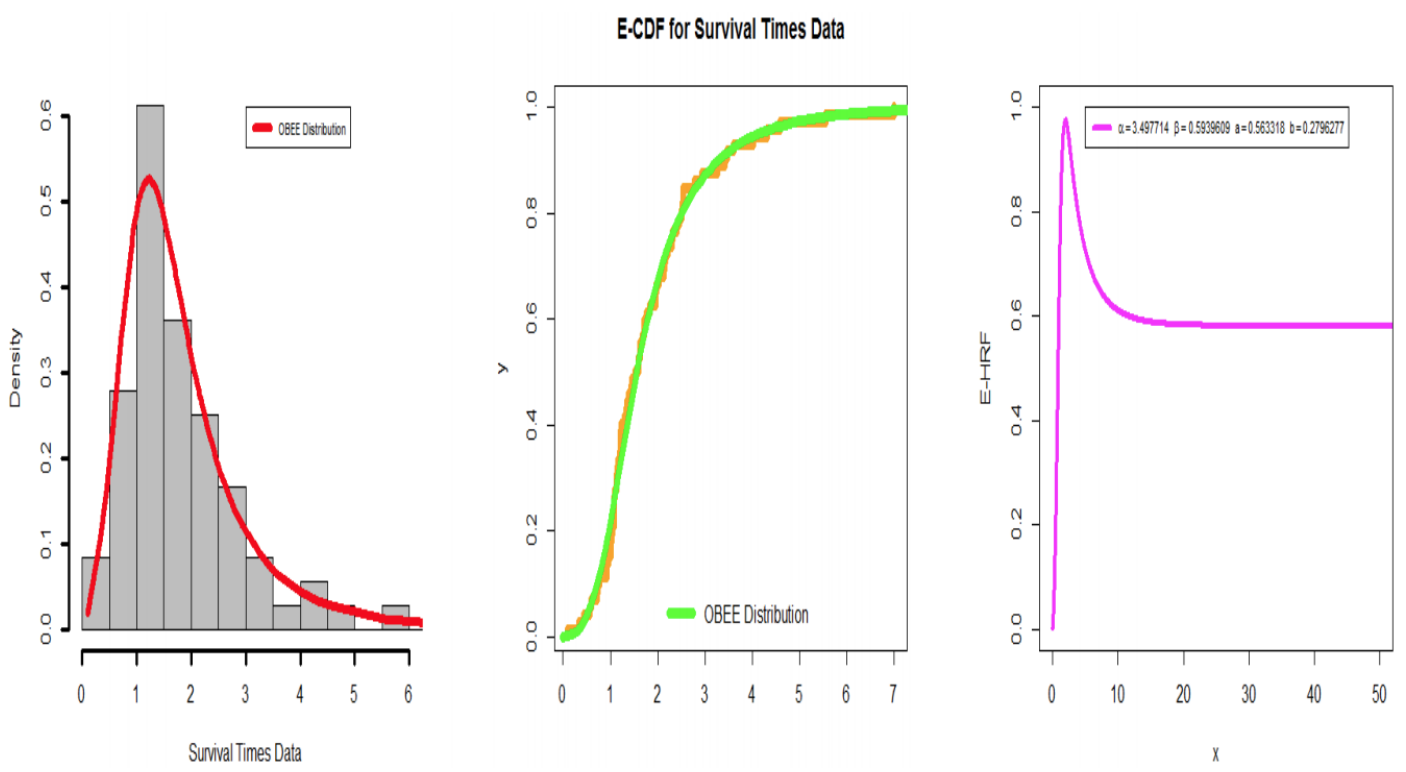

Figure 11. E-PDF, E-CDF and E-HRF for survival times data.

1 " or more than 1. Some bivariate and multivariate OBEE type model have been also derived. Estimation of OBEE parameters is performed by maximum likelihood estimation method. We assessed the performance of the maximum likelihood method. The assessment can be performed graphically via the biases and mean squared errors. The usefulness and flexibility of the new distribution is illustrated by means of two real data sets. The new model is much better than many other competitive models in modeling relief times and survival times data sets according to the Akaike Information Criterion, the Consistent Akaike Information Criterion, the Hannan-Quinn Information Criterion, the Bayesian Information Criterion, the Cramér-Von Mises, the Anderson-Darling statistics. As a future work, we can apply the Bagdonavičius-Nikulin goodness-of-fit test, 
Kaplan-Meier Survival Plot

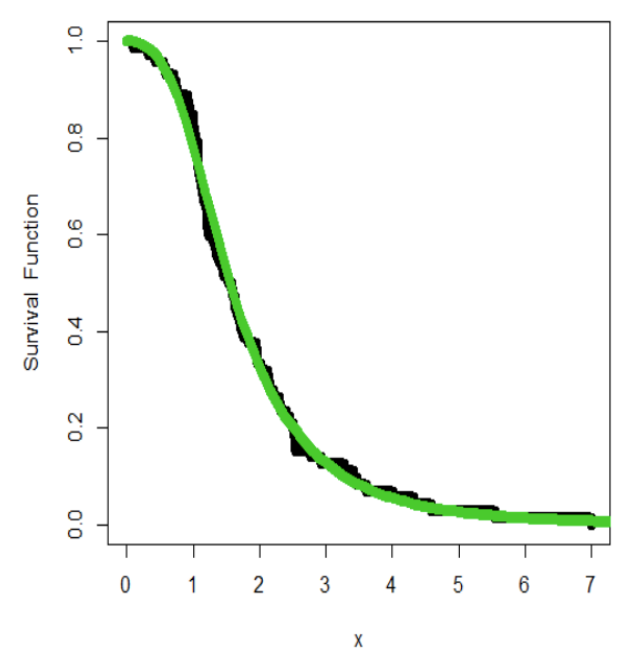

P.P plot (Survival Times)

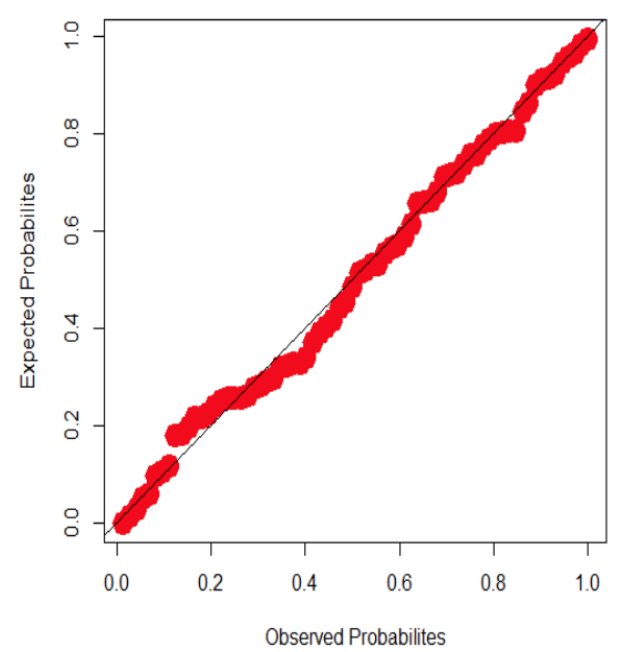

Figure 12. Kaplan-Meier survival plot and P-P plot for survival times data.

modified Bagdonavičius-Nikulin goodness-of-fit test, Nikulin-Rao-Robson goodness-of-fit test and modified Nikulin-Rao-Robson goodness-of-fit test to our new model (see Goual et al. ([27], [28] and [29]) and Yadav et al. [62] for more details). Characterization results and regression modeling can be derived based on OBEE model (see [9], [10], [11] and [12]for more details).

\section{REFERENCES}

1. Abouelmagd, T. H. M., Hamed, M. S. and Yousof, H. M. (2019a). Poisson Burr X Weibull distribution. Journal of Nonlinear Sciences \& Applications (JNSA), 12(3), 173-183.

2. Abouelmagd, T. H. M., Hamed, M. S., Almamy, J. A., Ali, M. M., Yousof, H. M. and Korkmaz, M. C. (2019b). Extended Weibull log-logistic distribution. Journal of Nonlinear Sciences and Applications, 12(8), 523-534.

3. Al-Babtain, A. A. Elbatal, I. and Yousof, H. M. (2020). A new flexible three-parameter model: properties, clayton copula, and modeling real data, Symmetry, 12, 1-17. doi:10.3390/sym12030440

4. Ali, M. M., Yousof, H. M. and Ibrahim, M. (2021a). A new version of the generalized Rayleigh distribution with copula, properties, applications and different methods of estimation. Optimal Decision Making in Operations Research \& Statistics: Methodologies and Applications, VOL 1, 1-25.

5. Ali, M. M., Ibrahim, M. and Yousof, H. M. (2021b). Expanding the Burr X model: properties, copula, real data modeling and different methods of estimation. Optimal Decision Making in Operations Research \& Statistics: Methodologies and Applications, VOL 1, 26-49.

6. Alizadeh, M., Ghosh, I., Yousof, H. M., Rasekhi, M. and Hamedani, G. G. (2017). The generalized odd generalized exponential family of distributions: properties, characterizations and applications. Journal of Data Science, 15(3), 443-465.

7. Alizadeh, M., Rasekhi, M., Yousof, H. M., Ramires, T. G. and Hamedani, G. G. (2018). Extended exponentiated NadarajahHaghighi model: Mathematical properties, characterizations and applications. Studia Scientiarum Mathematicarum Hungarica, 55(4), 498-522.

8. Alizadeh, M., Yousof, H. M., Jahanshahi, S. M. A., Najibi, S. M. and Hamedani, G. G. (2020). The transmuted odd loglogistic-G family of distributions. Journal of Statistics and Management Systems, 1-27.

9. Altun, E., Yousof, H. M. and Hamedani G. G. (2018). A Flexible Extension of Generalized Half-Normal Distribution: Characterizations and Regression Models. International Journal of Applied Mathematics and Statistics, 57(3), 27-49.

10. Altun, E., Yousof, H. M. and Hamedani G. G. (2018). A new flexible extension of the generalized half-normal lifetime model with characterizations and regression modeling. Bulletin of Computational Applied Mathematics, 6(1), 83-115.

11. Altun, E., Yousof, H. M. and Hamedani, G. G. (2018). A new generalization of generalized half-normal distribution: properties and regression models. Journal of Statistical Distributions and Applications, 5(1), 7.

12. Altun, E., Yousof, H. M. and Hamedani, G. G. (2018). A new log-location regression model with influence diagnostics and residual analysis. Facta Universitatis, Series: Mathematics and Informatics, 33(3), 417-449. 
13. Aryal, G. R., Ortega, E. M., Hamedani, G. G. and Yousof, H. M. (2017). The Topp-Leone generated Weibull distribution: regression model, characterizations and applications. International Journal of Statistics and Probability, 6(1), 126-141.

14. Aryal, G. R. and Yousof, H. M. (2017). The exponentiated generalized-G Poisson family of distributions. Stochastics and Quality Control, 32(1), 7-23.

15. Aslam, M., Kundu, D., Ahmad, M. (2010). Time truncated acceptance sampling plans for generalized exponential distribution. J. Appl. Stat. 37, 555-566.

16. Bhatti, F. A., Hamedani, G. G., Yousof, H. M. and Ali, A. (2021). On the Burr III-Moment Exponential Distribution. Thailand statistician, forthcomming.

17. Bjerkedal, T. (1960). Acquisition of resistance in Guinea pigs infected with different doses of virulent tubercle bacilli. American Journal of Hygiene, 72, 130-148.

18. Chesneau, C. and Yousof, H. M. (2021). On a special generalized mixture class of probabilistic models. Journal of Nonlinear Modeling and Analysis, 3(1), 71-92.

19. Cordeiro, G. M., Alizadeh, M., Ozel, G., Hosseini, B., Ortega, E. M. M. and Altun, E. (2017). The generalized odd log-logistic family of distributions: properties, regression models and applications. Journal of Statistical Computation andSimulation, 87(5), 908-932.

20. Elgohari, H. and Yousof, H. M. (2020a). A Generalization of Lomax Distribution with Properties, copula and Real Data Applications. Pakistan Journal of Statistics and Operation Research, 16(4), 697-711. https://doi.org/10.18187/pjsor.v16i4.3260

21. Elgohari, H. and Yousof, H. M. (2020b). New Extension of Weibull Distribution: copula, Mathematical Properties and Data Modeling. Statistics, Optimization \& Information Computing, 8(4), 972-993. https://doi.org/10.19139/soic-2310-5070-1036

22. El-Morshedy, M., Alshammari, F. S., Hamed, Y. S., Eliwa, M. S. and Yousof, H. M. (2021). A New Family of Continuous Probability Distributions. Entropy, 23, 194. https://doi.org/10.3390/e23020194

23. Elsayed, H. A. and Yousof, H. M. (2019). A new Lomax distribution for modeling survival times and taxes revenue data sets. Journal of Statistics and Applications, forthcoming.

24. Elsayed, H. A. H. and Yousof, H. M. (2019). The Burr X Nadarajah Haghighi distribution: statistical properties and application to the exceedances of flood peaks data. Journal of Mathematics and Statistics, 15, 146-157.

25. Elsayed, H. A. H. and Yousof, H. M. (2020). The generalized odd generalized exponential Fréchet model: univariate, bivariate and multivariate extensions with properties and applications to the univariate version. Pakistan Journal of Statistics and Operation Research, 529-544.

26. Farlie, D. J. G. (1960). The performance of some correlation coecents cients for a general bivariate distribution. Biometrika, 47, 307-323.

27. Goual, H. and Yousof, H. M. (2020). Validation of Burr XII inverse Rayleigh model via a modified chi-squared goodness-of-fit test. Journal of Applied Statistics, 47(3), 393-423.

28. Goual, H., Yousof, H. M. and Ali, M. M. (2019). Validation of the odd Lindley exponentiated exponential by a modified goodness of fit test with applications to censored and complete data. Pakistan Journal of Statistics and Operation Research, $745-771$.

29. Goual, H., Yousof, H. M. and Ali, M. M. (2020). Lomax inverse Weibull model: properties, applications, and a modified Chi-squared goodness-of-fit test for validation. Journal of Nonlinear Sciences \& Applications (JNSA), 13(6), 330-353.

30. Gross, J. and Clark, V. A. (1975). Survival Distributions: Reliability Applications in the Biometrical Sciences, John Wiley, New York, USA.

31. Gumbel, E. J. (1961). Bivariate logistic distributions. Journal of the American Statistical Association, 56(294), 335-349.

32. Gumbel, E. J. (1960) Bivariate exponential distributions. Journ. Amer. Statist. Assoc., 55, 698-707.

33. Hamedani, G. G., Rasekhi, M., Najibi, S., Yousof, H. M. and Alizadeh, M. (2019). Type II general exponential class of distributions. Pakistan Journal of Statistics and Operation Research, 15(2), 503-523.

34. Hamedani, G. G., Yousof, H. M., Rasekhi, M., Alizadeh, M. and Najibi, S. M., (2018). Type I general exponential class of distributions. Pakistan Journal of Statistics and Operation Research, 14(1), 39-55.

35. Ibrahim, M., Altun, E., Goual, H., and Yousof, H. M. (2020). Modified goodness-of-fit type test for censored validation under a new Burr type XII distribution with different methods of estimation and regression modeling. Eurasian Bulletin of Mathematics, 3(3), 162-182.

36. Ibrahim, M., Altun, E. and Yousof, H. M. (2020). A new distribution for modeling lifetime data with different methods of estimation and censored regression modeling. Statistics, Optimization \& Information Computing, 8(2), 610-630.

37. Mohamed, M. I., Handique, L., Chakraborty, S., Butt, N. S. and Yousof, M. H. (2021). A New Three-parameter Xgamma Fréchet Distribution with Different Methods of Estimation and Applications. Pakistan Journal of Statistics and Operation Research, 17(1), 291-308. https://doi.org/10.18187/pjsor.v17i1.2887

38. Ibrahim, M., Mohammed, W. and Yousof, H. M. (2020). Bayesian and Classical Estimation for the One Parameter Double Lindley Model. Pakistan Journal of Statistics and Operation Research, 409-420.

39. Ibrahim, M. Sharma, V. K., Korkmaz, M.C., Ali, M. M., Chesneau, C. and Yousof, H. M. (2020). A Generalization of Lindley Distribution: Copula, Properties and Different Methods of Estimation. MATHSTATICA. VOL1, 1-26.

40. Ibrahim, M., Yadav, A. S., Yousof, H. M., Goual, H. and Hamedani, G. G. (2019). A new extension of Lindley distribution: modified validation test, characterizations and different methods of estimation. Communications for Statistical Applications and Methods, 26(5), 473-495.

41. Ibrahim, M. and Yousof, H. M. (2020). Transmuted Topp-Leone Weibull lifetime distribution: Statistical properties and different method of estimation. Pakistan Journal of Statistics and Operation Research, 501-515.

42. Khalil, M. G., Hamedani, G. G., \& Yousof, H. M. (2019). The Burr X exponentiated Weibull model: Characterizations, mathematical properties and applications to failure and survival times data. Pakistan Journal of Statistics and Operation Research, 141-160.

43. Karamikabir, H., Afshari, M., Yousof, H. M., Alizadeh, M. and Hamedani, G. (2020). The Weibull Topp-Leone Generated Family of Distributions: Statistical Properties and Applications. Journal of The Iranian Statistical Society, 19(1), 121-161. 
44. Korkmaz, M. C., Altun, E., Yousof, H. M. and Hamedani, G. G. (2020). The Hjorth's IDB Generator of Distributions: Properties, Characterizations, Regression Modeling and Applications. Journal of Statistical Theory and Applications, 19(1), 59-74.

45. Korkmaz, M. C. and Yousof, H. M. (2017). The one-parameter odd Lindley exponential model: mathematical properties and applications. Stochastics and Quality Control, 32(1), 25-35.

46. Korkmaz, M. C., Yousof, H. M. and Hamedani, G. G. (2018). The exponential Lindley odd log-logistic-G family: properties, characterizations and applications. Journal of Statistical Theory and Applications, 17(3), 554-571.

47. Korkmaz, M. C., Yousof, H. M., Rasekhi, M. and Hamedani, G. G. (2018). The Odd Lindley Burr XII Model: Bayesian Analysis, Classical Inference and Characterizations. Journal of Data Science, 16(2), 327-353.

48. Kundu, D. and Pradhan, B. (2009). Bayesian inference and life testing plans for generalized exponential distribution. Sci. China Ser. A, Math. 52, 1373-1388.

49. Mansour, M. M., Ibrahim, M., Aidi, K., Shafique Butt, N., Ali, M. M., Yousof, H. M. and Hamed, M. S. (2020a). A New Log-Logistic Lifetime Model with Mathematical Properties, copula, Modified Goodness-of-Fit Test for Validation and Real Data Modeling. Mathematics, 8(9), 1508.

50. Mansour, M. M., Butt, N. S., Ansari, S. I., Yousof, H. M., Ali, M. M. and Ibrahim, M. (2020b). A new exponentiated Weibull distribution's extension: copula, mathematical properties and applications. Contributions to Mathematics, 1 (2020) 57-66. DOI: $10.47443 / \mathrm{cm} .2020 .0018$

51. Mansour, M., Korkmaz, M. C., Ali, M. M., Yousof, H. M., Ansari, S. I. and Ibrahim, M. (2020c). A generalization of the exponentiated Weibull model with properties, copula and application. Eurasian Bulletin of Mathematics, 3(2), 84-102.

52. Mansour, M., Rasekhi, M., Ibrahim, M., Aidi, K., Yousof, H. M. and Elrazik, E. A. (2020d). A New Parametric Life Distribution with Modified Bagdonavičius-Nikulin Goodness-of-Fit Test for Censored Validation, Properties, Applications, and Different Estimation Methods. Entropy, 22(5), 592.

53. Mansour, M., Yousof, H. M., Shehata, W. A. and Ibrahim, M. (2020e). A new two parameter Burr XII distribution: properties, copula, different estimation methods and modeling acute bone cancer data. Journal of Nonlinear Science and Applications, 13(5), 223-238.

54. Mansour, M. M., Butt, N. S., Yousof, H. M., Ansari, S. I. and Ibrahim, M. (2020f). A Generalization of Reciprocal Exponential Model: Clayton copula, Statistical Properties and Modeling Skewed and Symmetric Real Data Sets. Pakistan Journal of Statistics and Operation Research, 16(2), 373-386.

55. Merovci, F., Alizadeh, M., Yousof, H. M. and Hamedani, G. G. (2017). The exponentiated transmuted-G family of distributions: theory and applications. Communications in Statistics-Theory and Methods, 46(21), 10800-10822.

56. Merovci, F., Yousof, H. M. and Hamedani, G. G. (2020). The Poisson Topp Leone Generator of Distributions for Lifetime Data: Theory, Characterizations and Applications. Pakistan Journal of Statistics and Operation Research, 16(2), 343-355.

57. Morgenstern, D. (1956). Einfache beispiele zweidimensionaler verteilungen. Mitteilingsblatt fur Mathematische Statistik, 8 , 234-235.

58. Pougaza, D. B. and Djafari, M. A. (2011). Maximum entropies copulas. Proceedings of the 30th international workshop on Bayesian inference and maximum Entropy methods in Science and Engineering, 329-336.

59. Salah, M. M., El-Morshedy, M., Eliwa, M. S. and Yousof, H. M. (2020). Expanded Fréchet Model: Mathematical Properties, copula, Different Estimation Methods, Applications and Validation Testing. Mathematics, 8(11), 1949.

60. Yousof, H. M., Ali, M. M., Goual, H. and Ibrahim. M. (2021). A new reciprocal Rayleigh extension: properties, copulas, different methods of estimation and modified right censored test for validation, STATISTICS IN TRANSITION new series, forthcoming.

61. Yadav, A. S., Altun, E. and Yousof, H. M. (2019). Burr-Hatke Exponential Distribution: A Decreasing Failure Rate Model, Statistical Inference and Applications. Annals of Data Science, 1-20.

62. Yadav, A. S., Goual, H., Alotaibi, R. M., Ali, M. M. and Yousof, H. M. (2020). Validation of the Topp-Leone-Lomax model via a modified Nikulin-Rao-Robson goodness-of-fit test with different methods of estimation. Symmetry, 12(1), 57.

63. Yousof, H. M., Butt, N. S., Alotaibi, R. M., Rezk, H., Alomani, G. A. and Ibrahim, M. (2019). A new compound Fréchet distribution for modeling breaking stress and strengths data. Pakistan Journal of Statistics and Operation Research, 15(4), $1017-1035$

64. Yousof, H. M., Hamedani, G. G. and Ibrahim, M. (2020). The Two-parameter Xgamma Fréchet Distribution: Characterizations, copulas, Mathematical Properties and Different Classical Estimation Methods. Contributions to Mathematics, 2 (2020), 32-41.

65. Yousof, H. M., Korkmaz, M. C. and Sen, S. (2019). A new two-parameter lifetime model. Annals of Data Science, 1-16.

66. Zheng, G. (2002). On the fisher information matrix in type II censored data from the exponentiated exponential family. Biom. J. 44, 353-357.

67. Zheng, G. and Park, S. (2004). A note on time savings in censored life testing. J. Stat. Plan. Inference 124, 289-300. 